\title{
1 A plant-specific syntaxin-6 protein contributes to the intracytoplasmic route for
}

\section{2 begomoviruses}

4 Bianca Castro Gouveia-Mageste ${ }^{1 \&}$, Laura Gonçalves Costa Martins ${ }^{1 \&}$, Maximiller Dal-Bianco ${ }^{1,2}$,

5 João Paulo Batista Machado ${ }^{1,3}$, José Cleydson Ferreira da Silva ${ }^{1}$, Alice Y Kim ${ }^{4}$, Junshi Yazaki ${ }^{4,5}$

6 Anésia Aparecida dos Santos ${ }^{1,6}$, Joseph R Ecker $^{4},{ }^{7}$ and Elizabeth Pacheco Batista Fontes ${ }^{1,2 *}$

$8{ }^{1}$ National Institute of Science and Technology in Plant-Pest Interactions, Bioagro, Universidade

9 Federal de Viçosa, Viçosa, Minas Gerais, Brazil

$10{ }^{2}$ Department of Biochemistry and Molecular Biology, Universidade Federal de Viçosa, Viçosa,

11 Minas Gerais 36570-000, Brazil

$12{ }^{3}$ Agronomy Institute, Universidade Federal de Viçosa, Campus Florestal, Florestal, Minas Gerais, 13 Brazil

$14{ }^{4}$ Genomic Analysis Laboratory, Plant Biology Laboratory, Salk Institute for Biological Studies, 15 La Jolla, CA 92037, USA

$16{ }^{5}$ RIKEN Center for Integrative Medical Sciences, Yokohama City, Kanagawa 230-0045, Japan

$17{ }^{6}$ Departament of General Biology, Universidade Federal de Viçosa, Viçosa, Minas Gerais, Brazil

$18{ }^{7}$ Howard Hughes Medical Institute and Plant Biology Laboratory, The Salk Institute of Biological

19 Studies, La Jolla, United States of America

$21 *$ Corresponding author: bbfontes@ufv.br

22 \&These authors contributed equally to this work

23 Running title: NISP in begomovirus intracellular transport 


\section{Abstract}

26 Due to limited free diffusion in the cytoplasm, viruses must use active transport mechanisms to

27 move intracellularly. Nevertheless, how the plant ssDNA begomoviruses hijacks the host

28 intracytoplasmic transport machinery to move from the nucleus to the plasmodesmata remains

29 enigmatic. Here, we identified nuclear shuttle protein (NSP)-interacting proteins from Arabidopsis

30 by probing a protein microarray and demonstrated that the Cabbage leaf curl virus (CabLCV)

31 NSP, a facilitator of the nucleocytoplasmic trafficking of viral (v)DNA, interacts with an

32 endosomal vesicle-localized plant-specific syntaxin-6 protein, designated NSP-interacting

33 syntaxin-6 domain-containing protein (NISP) in planta. NISP displays a pro-viral function, but

34 not the syntaxin-6 paralog AT2G18860 that failed to interact with NSP. Consistent with these

35 findings, nisp-1 mutant plants were less susceptible to begomovirus infection, a phenotype

36 reversed by NISP complementation. NISP-overexpressing lines accumulated higher levels of viral

37 DNA than wild-type. Furthermore, NISP interacted with NIG, an NSP-interacting GTPase

38 involved in NSP-vDNA nucleocytoplasmic translocation. The NISP-NIG interaction was

39 enhanced by NSP. We also showed that NISP associates with vDNA and might assemble a NISP-

40 NIG-NSP-vDNA- complex. NISP may function as a docking site for recruiting NIG and NSP into

41 endosomes, providing a mechanism for the intracytoplasmic translocation of the NSP-vDNA

42 complex towards to and from the cell periphery.

\section{Author Summary}

45 As viruses must use an active and directed intracellular movement, they hijack the intracellular

46 host transport system for their own benefits. Therefore, the identification of interactions between 
47 host proteins and begomovirus movement proteins should target the intracellular transport

48 machinery. This work focused on the identification of these protein-protein interactions; it

49 addressed the molecular bases for the intracellular transport of begomoviruses. We used a protein

50 microarray to identify cellular partners for the movement protein (MP) and the viral nuclear shuttle

51 protein (NSP), which is a facilitator of the nucleocytoplasmic trafficking of viral (v)DNA. We

52 identified relevant protein-protein interaction (PPI) hubs connecting host and viral proteins. We

53 revealed a novel NSP-interacting protein, which functions in the intracytoplasmic transport of

54 proteins and DNA from begomoviruses and was designated NSP-interacting syntaxin domain-

55 containing protein (NISP). Our data suggest an intracellular route connecting the release of newly

56 synthesized begomoviral DNA in the cytosol with the cell surface. Resolving viral DNA-host

57 protein complexes led to the identification of a novel class of components of the cell machinery

58 and a representative member, NISP, that functions as a susceptibility gene against begomoviruses.

59 As geminiviruses pose a severe threat to agriculture and food security, this recessive gene can now

60 be exploited as a target for engineering resistance by gene editing in crops. 


\section{Introduction}

As obligate intracellular parasites, viruses must enter the host cells and hijack the cell machinery to complete their life cycle. Independent on the molecular mechanisms for virus entry

67 into cells, which differ entirely between animal and plant viruses, once in the cytoplasm, both plant

68 and animal viral genomes move intracellularly to specific cellular sub-compartments for

69 replication, transcription or encapsidation [1,2]. Due to the limitation of free diffusion in the

70 cytoplasm, viruses have invoked the endogenous host movement mechanisms for an active and

71 directed intracytoplasmic translocation. In fact, almost all viruses are capable of subverting the

72 microtubule transport system to some extent to replicate and spread. Plant viruses must also use

73 an active mechanism to move the viral genome from the replication site to the plasmodesmata,

74 which are intercellular cytoplasmic bridges between plant cells [2,3]. To promote this typical cell-

75 to-cell movement, plant viruses have evolved movement proteins that increase the size exclusion

76 limit of the plasmodesmata and actively promote the viral nucleic acid translocation into

77 uninfected, adjacent cells [4]. In the case of viruses that replicate in the nuclei of infected cells,

78 they have to be directed to the nuclear periphery, followed by nuclear entry. Likewise, the newly-

79 synthesized viral proteins and viral genomes must translocate throughout the cytoplasm during

80 exit. In animal cells, the retroviruses and DNA viruses deploy the microtubule motor system and

81 microtubule cytoskeleton to move viral proteins and genomes into the nucleus and back to the

82 plasma membrane $[1,5]$. Geminiviruses are typical examples of plant DNA viruses that replicate

83 in the nuclei of infected cells, but the underlying mechanisms for the intracytoplasmic movement

84 of viral proteins and viral DNA (vDNA) are far less understood.

85 Geminiviruses are circular single-stranded (ss) DNA viruses, which replicate via double- 
86 stranded (ds) DNA intermediates in the nucleus of infected cells, infect a broad spectrum of

87 economically important crops and cause significant yield losses worldwide [6]. The Geminiviridae

88 family includes nine genera, but approximately $75 \%$ of the identified species belong to the genus

89 Begomovirus, which are whitefly-transmitted, infect dicotyledonous species, and display a genome

90 configuration that can be either monopartite or bipartite $[7,8]$. The genomic components of the

91 bipartite begomoviruses are designated DNA-A and DNA-B. The DNA-A encodes proteins

92 required for viral replication (AC1 or Rep, $\mathrm{REn}$ or $\mathrm{AC} 3)$, transactivation of viral genes (AC2 or

$93 \mathrm{TRaP})$, encapsidation of viral genome (AV1 or CP) and suppression of host defenses (AC4 and

94 TrAP). DNA-B encodes the nuclear shuttle protein (NSP or BV1) and the movement protein (MP

95 or BC1) involved in intracellular and cell-to-cell movement of vDNA.

96 Although the role of MP-NSP complex formation in mediating intracellular and

97 intercellular transport of begomoviruses is widely accepted, the molecular mechanisms underlying

98 the action of these viral proteins at individual stages of the intracellular and intercellular movement

99 are still debated. Some studies indicated that NSP from Bean dwarf mosaic virus (BDMV) and

100 Abutilon mosaic virus (AbMV) complexes with ss-vDNA and ds-vDNA presumably in the nuclei

101 of infected cells, whereas NSP from Squash leaf curl virus (SLCV) binds only ss-vDNA in vitro

$102[9,10,11]$. NSP also interacts with MP in the cytoplasm [12], which promotes the directionality

103 of virus translocation to the cell surface $[12,13]$. The mechanism for vDNA exit from the nucleus

104 remains unsolved. It has been conceptually accepted that NSP facilitates the nuclear exit of newly-

105 replicated vDNA via nuclear pores [10, 14-17]. However, the interactions with the host nuclear

106 transport machinery have not been documented. A piece of this puzzle was obtained with the

107 identification of an NSP-interacting GTPase (NIG), which interacts with NSP at the cytosolic side

108 of the nuclear pore complex and facilitates the release of NSP-vDNA complex from the nuclear 
envelope into the cytosol $[12,18]$. At this step, it has not been resolved whether NSP-vDNA is

110 free in the cytosol or is transported via vesicles at their protoplasmic leaflet to the periphery.

111 Consistent with this transport function, the cytosolic NIG (i) accumulates around the nuclear

112 envelope, (ii) promotes the translocation of NSP-vDNA from the nucleus to the cytoplasm, (iii)

113 functions as a pro-viral factor during begomovirus infection and (iv) shares structural features and

114 transport properties with the human Rev-Interacting Protein (hRIP), which has been shown to be

115 involved in the release of HIV-1 RNAs from the nuclear periphery to the cytoplasm $[12,18,19]$.

116 Histone H3 has been shown to bind NSP and MP from BDMV as an additional component of the 117 vDNA-protein complex that traffics intracellularly and intercellularly [20]. A nuclear

118 acetyltransferase (AtNSI) interacts with the Cabbage leaf curl virus (CabLCV) NSP and may 119 regulate the nuclear export of ss-vDNA-NSP via acetylation of CP and histones [21, 22]. Other 120 NSP-interacting partners include the receptor-like kinases (RLKs) NSP-interacting kinase (NIK1) 121 and NSP-associated kinase (NsAK), which are not involved in intracellular movement of vDNA 122 but rather are implicated in antiviral immunity against begomoviruses [23-26].

123 For the cell-to-cell transport of vDNA, two distinct mechanisms, which basically differ in 124 the nature of the vDNA-protein complex translocated into adjacent cells, have been suggested [11, 125 27]. In the first mechanism, designated "relay race" model, NSP facilitates the translocation of 126 viral DNA from the nucleus to the cytoplasm and then is replaced by MP, which promotes the cell127 to-cell movement of vDNA via plasmodesmata $[10,14]$. The second mechanism represented by 128 the "couple-skating" model preconizes that MP facilitates the vDNA-NSP nucleocytoplasmic 129 trafficking and promotes the translocation of vDNA-NSP complex into the adjacent cells via 130 endoplasmic-reticulum-derived tubules induced by the viral infection [11-13, 18, 28-32]. More 131 recently, an alternative route for intracellular translocation and cell-to-cell spread of vDNA via 
132 chloroplasts and stromules has been proposed, which was based on experimental data of AbMV

133 MP interactions $(33,34)$. AbMV infection has been shown to induce the formation of a stromule

134 network interconnecting different chloroplasts, chloroplasts with nuclei, and expanding to the cell

135 periphery presumably at plasmodesmata [34]. This stromule network is stabilized by the plastid

136 chaperone cpHSC70, which has also been shown to interact with MP [33]. The interaction between

$137 \mathrm{MP}$ and cpHSC70 may bridge viral nucleoprotein complexes with stromules. Independent on the

138 cell-to-cell transport models, the underlying mechanisms of the intracytoplasmic retrograde and

139 anterograde movements of vDNA complexes remain to be determined. CabLCV and SLCV MPs

140 have been shown to interact with synaptotagmin A (SYTA), which is located in the endosomes of

141 plant cells and is required for the cell-to-cell trafficking of different MPs [35]. These findings

142 raised the hypothesis that distinct viral MPs promote an intracytoplasmic transport of vDNA-

143 complexes to plasmodesmata via an endocytic recycling pathway. Likewise, the AbMV MP has

144 been shown to interfere with microtubule assembly and stability when co-expressed in plant tissues

145 with a viral inducer of cell progression, which may suggest a microtubule-associated transit of MP

146 or begomoviruses to the plasmodesmata [36]. However, the host components of the vDNA

147 complex competent for intracytoplasmic translocation have not been identified, and the underlying

148 mechanism for intracytoplasmic trafficking of vDNA remains to be elucidated.

149 In this investigation, we used a protein microarray assay to identify MP- and NSP-host

150 protein-protein interactions (PPIs). From the NSP-MP-host PPI network map, we selected an

151 intracellular transport-associated protein, designated NSP-interacting syntaxin domain-containing

152 protein (NISP), as an NSP specific target, to gain further insight into the intracytoplasmic transport

153 of begomoviruses. We showed that NISP, which was located in motile vesicle-like structures,

154 many of which were targeted to the endosomes, interacted with NSP in vivo and displayed a pro- 
155 viral function. Furthermore, NISP also interacted with NIG, and the complex formed was enhanced

156 by the presence of viral NSP. In addition, vDNA might have been recruited into the NISP complex.

157 Our data indicate that NISP might be part of the vDNA complex that traffics intracellularly via 158 endosomes.

161 Results

163 Identification of Arabidopsis proteins interacting with in vitro transcribed and translated 164 MP and NSP

To search for the begomovirus movement proteins-Arabidopsis protein-protein interactions (PPIs), we used a nucleic acid programmable protein array (NAPPA)-based approach

167 [37], in which we interrogated 4600 in vitro synthesized ORFs from Arabidopsis for their capacity

168 to be targeted by MP and/or NSP from CabLCV. The in situ synthesized NAPPA protein 169 microarray has previously generated a transcription factor (TF)-NAPPA dataset, which has been 170 validated by pull-down assays and bimolecular fluorescence complementation (BiFC) assays in $N$.

171 benthamiana leaves using a subset of newly identified interactions [37]. NSP and MP were in vitro

172 transcribed and translated to probe this protein microarray (S1a and S1b Fig). A total of 40

173 candidate proteins were identified each for MP and NSP. The majority (35 proteins of 40) were

174 found to interact with both proteins, which was not surprising because MP itself interacts with

175 NSP and may be part of common multiprotein complexes as both participate in viral DNA 176 movement (S2 Fig). We found five new NSP-specific protein interactions (dark orange) and five 177 MP-specific protein interactions (light yellow), including a peptidyl-prolyl cis-trans isomerase 
178 family protein (AT1G73655) belonging to the same protein family as Pin4, which has been shown

179 previously to interact with MP (S1 Table) [36]. As previously identified NSP-interacting proteins

180 were absent in the protein array, the identification of known MP-specific interacting protein

181 contributed to validate our current data.

The protein-protein interactions between the viral proteins and the Arabidopsis proteins

183 were integrated into the protein-protein interactome experimentally determined for Arabidopsis

184 thaliana (BioGRID database and the Arabidopsis interactome database), using Cytoscape

185 software. This procedure identified the PPI network containing MP, NSP and all, directly and

186 indirectly, interacting host proteins (Fig 1). AT1G68185, an ubiquitin-like protein, which formed

187 a large hub (hub 20, degree 148), may represent a convergent targeting node among begomovirus

188 viral proteins as it was found to be a direct target of NSP and MP independently (S1 Table).

189 Furthermore, the ubiquitin-proteasome pathway (UPS) is targeted by many viruses to maintain

190 suitable levels of viral proteins and to induce, inhibit or modify ubiquitin (Ub)-related host proteins

191 [38]. Other MP-interacting proteins, including FKBP-like peptidyl-prolyl cis-trans isomerase

192 family protein (this work), chloroplast heat shock protein 70-1 [33] and synaptotagmin A [35],

193 which have been shown to assist the MP movement function, are connected to the AT1G68185

194 hub (hub 20), further supporting the notion that this hub may be biologically significant during

195 begomovirus infection.

To gain further insights into the cellular processes affected by NSP or MP, we performed

197 functional enrichment analyses of their direct and indirect interactors. Significantly enriched GO 198 terms (with $p$-value $<0.05$ ) were identified in all three categories, Biological Process, Molecular 199 Function, or Cellular Component ontology (S2 Table). Some over-represented GO terms in this 200 set of viral protein-interacting host proteins are consistent with NSP and MP function or 
201 localization. Consistent with their function as viral movement proteins, a subset of host interactors

202 was significantly over-represented under the transport activity term and protein binding term in

203 Molecular Function (S2 Table). Under the cellular component ontology, we observed an over-

204 representation of proteins under membrane-bounded organelle term, intracellular vesicle term, and

205 SNARE complex term, which may suggest an intracytoplasmic route for begomovirus proteins

206 and vDNA trafficking (S2 Table). Accordingly, two NSP-interacting proteins, AT5G23550 and

207 AT4G30240, form convergent hubs (17 and 19) enriched for membrane-bound proteins and

208 transport activity function (Fig 1, S1 Table). The NSP-interacting protein AT5G23550 (hub 17)

209 is annotated as a Got1/Sft2-like vesicle transport protein, which may be located to a late-Golgi

210 compartment and may be involved in the fusion of retrograde transport vesicles derived from an

211 endocytic compartment with the Golgi complex [39]. Likewise, AT4G30240 (hub 19) is a soluble

212 N-ethylmaleimide-sensitive factor attachment protein receptor (SNARE)-like protein harboring a

213 syntaxin-6 domain typical of proteins found in the endosomal transport vesicles [40].

214 Under the biological process ontology, the response to auxin term represented a

215 significantly enriched GO term, which has not been previously associated with begomovirus

216 infection (S2 Table). The NSP-MP-host PPI network uncovered two relevant hubs enriched for

217 auxin response-related proteins, which are represented by an NSP-MP general interaction-derived

218 hub (Fig 1, hub 9) and an NSP-specific host interaction-derived hub (hub 11). Remarkably, these

219 two viral protein hubs are interconnected to each other via interactions with proteins (in gray),

220 which are also over-represented under the auxin response term, forming a large auxin affected hub

221 target by begomovirus (S1 Table). This hub may represent an undocumented biological process

222 affected by begomovirus infection. The resulting PPI network map may provide a framework for 
223 future studies in detailing the begomovirus complex life cycle. One such example is described

224 here, as following.

225

226 A SNARE-like protein designated NSP-Interacting Syntaxin domain-containing Protein

227 (NISP), but not its paralog, interacts with NSP via the syntaxin domain

228 The NSP-MP-Host PPI network uncovered enriched GO terms linked to intracellular

229 vesicle and membrane-bound proteins, which may be associated with a mechanism for

230 intracytoplasmic translocation of viral proteins and vDNA. To pursue further with this possible

231 connection and to validate further the microarray data, we selected a new specific NSP-interacting

232 protein, the SNARE-like protein AT4G30240, which harbors a syntaxin-6 domain at the N-

233 terminus and a transmembrane segment at the C-terminus, for further analyses (Fig 2a). SNARE-

234 complexes drive the fusion of membrane-bound vesicles with target membranes required for

235 intracellular transport via the endosome system. We used the syntaxin domain superfamily as the

236 prototype for the identification of homologs in the genomes of Arabidopsis thaliana, Oryza sativa,

237 Drosophila melanogaster, Homo sapiens, and Saccharomyces cerevisiae. We then selected the

238 most related proteins to construct phylogenetic trees using Bayesian inference method (S3a Fig).

239 AT4G30240 was clustered in pair with AT2G18860 and formed a clade of syntaxin-6 domain-

240 containing proteins, which also included a third Arabidopsis paralog AT1G27700. This

241 AT4G30240-derived clade (in red), also designated Syntaxin-6-like subgroup, clustered only plant

242 proteins, carrying an N-terminal syntaxin-6-like domain and a C-terminal transmembrane

243 segment. AT4G30240 and AT2G18860 may be a result of genome duplication, as these syntaxin-

244 6-like proteins clustered in pair even in phylogenetic analyses that include proteins from other 
organisms, share a high degree of sequence conservation and display similar intron/genomic DNA configuration (https://www.arabidopsis.org/).

The remaining syntaxin-6-like proteins clustered together in a distinct clade that included 248 proteins from human, rice and the Arabidopsis AT1G28490, also named SYP61, a characterized trans-Golgi network (TGN)/endosome localized syntaxin [41-43]. This SYP61 second clade (in 250 orange) was designated target (t)-syntaxin-6/SNARE-like subgroup, as it included structurally

251 similar proteins carrying the N-terminal syntaxin-6 domain and also a t-SNARE coiled-coil 252 homology domain at the C-terminus.

254 SNARE superfamily, since it lacks the coiled-coil t-SNARE domain at the C-terminus, which is 255 responsible for vesicle fusions. A function of this subgroup has to be determined, yet. This 256 hypothesis was further confirmed in a phylogenetic analysis of syntaxin-6 domain-containing

257 proteins from Arabidopsis thaliana, Oriza sativa, Glycine max, Zea mays, Zostera marina, Homo

258 sapiens, Mus musculus, Bos taurus, Equus caballus, Danio rerio, and Drosophila melanogaster

259 using the full-length sequence of AT4G30240 as prototype (S3b Fig). The syntaxin-6 domain260 containing proteins form two distinct clades with high internal stability (100\%). The AT4G30240-

261 derived clade, syntaxin-6-like clade, includes only plant syntaxin-6-like proteins.

262 To complement the protein microarray data, we used the yeast two-hybrid system to 263 examine further the NSP-AT4G30240 interaction and compared the paralog AT2G18860 in our 264 assays, which was not present in the protein microarray. The second most closely related paralog, 265 AT1G28490, was present in the protein microarray but was not found to interact with NSP. Co266 expression of the full-length AT4G30240 (NISP) ORF fused to the activation domain (AD), and 267 NSP fused to the binding domain (BD) of GAL4 promoted histidine prototrophy (Fig 2b). The 
268 HIS3 marker gene was not activated in yeast cells cotransformed with the controls. In contrast,

269 AT2G18860 failed to interact with NSP in yeast, as neither AD-AT2G18860 nor BD-AT2G18860

270 promoted his prototrophy when cotransformed with the respective cognate BD-NSP or AD-NSP

271 (Fig 2c).

272 To map the NSP-interacting domain on NISP, we generated NISP deletion fragments and

273 assay for protein interactions by two-hybrid assay. The N-terminal NISP fragment extending up

274 to amino acid position 107 and containing an intact syntaxin-6 domain (NISP ${ }^{1-107}$, Fig 2a) was

275 sufficient to bind NSP in yeast (Fig 2d). The interaction of the truncated NISP ${ }^{1-107}$ with NSP was

276 specific because the HIS marker gene was not activated in yeast cells co-transformed with AD- or

277 BD-truncated NISP ${ }^{1-107}$ and the empty vectors (Fig 2d). In contrast, the NISP truncated versions,

278 comprising the NISP internal region, delimited by positions $105-200$ (NISP ${ }^{105-200}$; Fig 2e) or the

279 C-terminal portion (NISP ${ }^{200-300}$; Fig 2f), were not capable of recognizing NSP, confirming that

280 NISP binds NSP via its syntaxin-6 domain. Although the syntaxin-6 domains of NISP and

281 AT2G18860 are conserved (78.57\% identity), NSP interacts specifically with NISP in yeast (Fig

282 2b). Sequence alignment revealed an 8-amino acid deletion at the syntaxin-6 domain of

283 AT2G18860 as the most striking feature that may account for the differences in protein binding

284 specificity (S4 Fig).

285 Although NISP lacks a typical coiled-coil t-SNARE domain, it harbors a syntaxin-6

286 domain at the N-terminus and a predicted transmembrane segment at the C-terminus, which

287 suggests an endomembrane-bound localization (Fig 2a). Mammalian syntaxin-6 domain proteins

288 are localized in the trans-Golgi network (TGN) membranes and endosomal vesicles [44]. To

289 examine the subcellular localization of NISP, we transiently expressed AT4G30240 fused to a

290 green fluorescent protein (GFP) under the control of the native and constitutive promoters, in 
291 Nicotiana benthamiana leaves and analyzed the localization of the fluorescent signal by confocal

292 microscopy. NISP-GFP was predominantly localized in motile spherical structure, resembling

293 vesicles (Fig 3a, 3b; S1 Video). The paralog AT2G18860 fused to GFP appeared concentrated

294 predominantly in membrane-bound vesicles and plasma membrane (S5 Fig).

295

To examine more precisely the NISP localization, we performed co-localization assays,

296 using SYTA-mCherry as an endosome-associated marker [35], the plasma membrane-associated

297 molecular marker FLS2-mCherry [45], ER-, TGN- and tonoplast-associated molecular markers

298 constructed by Nelson et al. [46]. NISP-GFP did not co-localize with ER-mCherry- (Fig 3c,

299 asterisks indicate the field of the insets) and tonoplast (vac-mCherry)- (Fig 3e) associated

300 molecular markers. Likewise, NISP-containing vesicles did not co-localize with the plasma

301 membrane FLS2-mCherry (Fig 3f). In contrast, a small population of NISP-GFP-containing

302 vesicles co-localized with the TGN-associated molecular marker (Fig 3d) and a large fraction of

303 NISP-GFP-containing vesicles precisely co-localized with the endosomal protein SYTA-mCherry

304 (Fig 3g). Because many of the NISP-labeled vesicles were associated with the plasma membrane,

305 we examined whether the NISP-associated vesicle membrane was derived from the plasma

306 membrane by staining NISP-GFP-expressing cells with FM4-64, a marker for plasma membrane

307 internalization that labels endosomes [47]. In addition to dye-labeled plasma membranes, some

308 vesicles showed also NISP-GFP fluorescence (Fig 3H, arrows). Collectively, these results indicate

309 that NISP localizes in TGN/early endosome vesicles.

311 NISP interacts with NSP in intracellular vesicles

We next examined whether NISP interacts with NSP in vivo using the bimolecular

313 fluorescence complementation (BiFC) assay. The formation of NISP/NSP complexes occurred 
314 atvesicles of $N$. benthamiana epidermal cells independent of the orientation of the NISP fusions

315 (N-terminus or C-terminus of YFP; Fig 4a), and the reconstituted fluorescent signal was much

316 higher than that of the background (control panels with combinations of the protein fusions with

317 empty vectors). The reconstituted fluorescent signal was co-labeled by FM4-64, indicating that the

318 complex was formed in endosomal vesicles derived from the plasma membrane (Fig 4b, see

319 arrows). We further demonstrated that NISP and NSP interacted in vivo using co-

320 immunoprecipitation assays. Both NISP-GFP and NSP-6HA, were co-immunoprecipitated from

321 co-infiltrated extracts using anti-GFP antibody, demonstrating an association of NISP-GFP with

322 NSP-6HA fusions (Fig 4c). Switching the protein tags and including MP did not alter the results

323 because NISP-6HA also associated with NSP-GFP in co-infiltrated extracts (Fig 4d). Co-

324 expression of MP in the assay was used to mimic infection, since CabLCV MP promotes the

325 directionality of NSP to the cell periphery $(12,13)$. The Co-IP results also demonstrated that GFP

326 alone did not interact with either NSP-6HA or NISP-6HA, demonstrating the specificity of the

327 interactions between the viral protein and NISP.

329 NISP, but not its paralog AT2G18860, displays a pro-viral function

The biological relevance of the NSP-NISP interactions in begomovirus infection was

331 examined using several different approaches. We first analyzed the expression of NISP from

332 publically available RNA-seq data, which showed that NISP was induced by begomovirus

333 infection [26]. Quantitative RT-PCRs showed that NISP RNAs, but not the RNA from the NISP

334 paralog AT2G18860, were induced by the begomovirus infection (S6 Fig). We then identified a

335 T-DNA insertion mutant in the NISP gene (nisp-1) and the AT2G18860 gene (at2gl8860-1). The

336 NISP or AT2G18860 transcript levels in the respective T-DNA insertion mutant lines were much 
337 lower than in Col-0, indicating that nisp-1 and at2g18860-1 may be loss-of-function mutant lines

338 (Fig 5a, 5c). These mutant lines were transformed with NISP-GFP or AT2G18860-GFP constructs

339 to analyze a complementation of the defects in three independent lines each (Fig 5a, 5b, nisp-

340 1/NISP lines, and at2g18860-1/AT2G18860 lines). We also prepared transgenic lines

341 overexpressing NISP-GFP and AT2G18860-GFP, under the control of the 35S constitutive

342 promoter. The respective transcript (Fig 5a, 5c) levels were higher in NISP and AT2G18860 plants

343 than in Col-0 and accumulation of the encoding proteins was determined by immunoblotting with

344 an anti-GFP antibody (Fig 5b, 5d). The knockout and overexpressing lines were phenotypically

345 indistinguishable from Col-0 (S8 Fig).

We next examined whether NISP and AT2G18860 participate in begomovirus infection.

347 The knockout lines (nisp-1 and at2g18860-1), complemented lines (nisp-1/NISP and at2g18860-

348 1/AT2G18860), NISP-overexpressing lines and Col-0 were inoculated with infectious clones of

349 CabLCV and the course of infection was monitored by visualization of symptom development and

350 PCR diagnosis. The accumulation of viral DNA was quantified by qPCR (Fig 5).

351 The nisp-1 knockout line displayed attenuated symptoms (Fig 5e, S7 Fig), delayed course

352 of infection (Fig 5f), and lower viral DNA as compared to Col-0 (Fig 5g). Expression of NISP-

353 GFP in nisp-1 restored the wild type susceptibility to begomovirus infection in all three

354 independently transformed lines (nisp-1/NISP), confirming that the reduced susceptibility

355 displayed by the nisp-1 line was indeed due to loss of NISP function. In contrast, NISP

356 overexpression conferred enhanced susceptibility to begomovirus infection; the progress of

357 infection was accelerated (Fig 5f), and viral DNA load was significantly higher in the NISP-

358 overexpressing lines than in Col-0 and nisp-1 lines (Fig 5g). We predicted that if NISP-NSP

359 binding was the basis for the NISP pro-viral function, the closest paralog of NISP, AT2G18860, 
which harbors the syntaxin-6-like domain but does not interact with NSP, would not affect complemented lines, and an AT2G18860-overexpressing line displayed similar symptoms, course of infection and viral DNA load as Col-0, indicating that the presence or absence of the AT2G18860 gene does not change the begomovirus infection (Fig 5e, 5f, 5g, S7 Fig). These results further substantiated a specific pro-viral function of NISP rather than of syntaxin-6 like domain proteins in general.

NISP also interacts with NIG and complex formation is enhanced by viral NSP

There is a gap in our knowledge of how the vDNA-NSP complex, which binds to NIG in

370 the cytosolic side of the nuclear pores, traffics to the cell periphery. From the PPI network map,

371 we hypothesized that NIG could serve as a bridge to attach vDNA to the intracellular transport

372 system of plant cells. The integration of our data with the Arabidopsis interactome uncovered a

373 possible interaction between NIG and NISP, which was also shown here to bind specifically to

374 viral NSP. The interaction between NIG and NISP was first demonstrated by two-hybrid assay

375 (S9a Fig). The co-expression of the NISP ORF fused to BD and NIG ORF fused to AD from GAL4

376 promoted histidine prototrophy in medium supplemented with $2.5 \mathrm{mM} 3 \mathrm{AT}$, whereas co-

377 expression with empty vectors did not.

To examine whether NIG and NISP interact in vivo, we used BiFC and co-

379 immunoprecipitation assays. NIG has been shown to be unevenly distributed around the nuclear 380 envelope, and cell periphery (S9b and S9c Fig) $[25,48]$. In the BiFC analyses, nYFP-NIG as well 381 as cYFP-NISP (and vice-versa) were localized at the cell periphery (Fig 6a), probably at the NISP 382 endosomes, since the structures were co-stained by FM4-64 (Fig 6b; arrows). The fluorescent 
383 signal was barely detected in the controls (Fig 6a). The inclusion of NSP-6HA in the BiFC assay

384 increased the NISP-NIG complex formation, as judged by the enhanced intensity of the

385 reconstituted fluorescent signal, showing the fluorescent vesicles associated with the plasma

386 membrane (S10 Fig). To provide further evidence of the NSP-mediated enhancement of NIG-

387 NISP interaction, we used a quantitative co-immunoprecipitation assay (Fig 6, S9 Fig). We first

388 confirmed that NIG interacts with NISP in vivo (Fig 6c, S10d). The Co-IP results indicated that

389 NISP-GFP, but not GFP alone, interacted with NIG-HA. Likewise, NIG-GFP, but not GFP alone,

390 interacted with NISP-HA. NSP promoted a stronger association of NISP-GFP with NIG-6HA, as

391 judged by the amount of co-immunoprecipitated NIG by anti-GFP in the presence of co-expressed

392 GST-NSP (Fig 6d, 6e). Likewise, GST-NSP enhanced NISP-6HA-NIG-GFP complex formation

393 (S9e and S9f Fig). These results indicate that NSP/NIG and NISP might form a multiprotein

394 complex at endosomes. Syntaxin-6 domain-containing proteins have been shown to participate in

395 the endosome-plasma membrane route of protein cargos.

397 NISP complexes viral DNA in vivo

The current model of vDNA intracellular translocation informs that NSP binds to nascent

399 viral DNA in the nucleus, facilitates its translocation to the cytosol via nuclear pores and moves

400 vDNA intracytoplasmically to the cell periphery, where MP mediates the intercellular movement

401 of vDNA-NSP or vDNA-MP via plasmodesmata. Because NSP also binds to NISP in vesicle-like

402 structures, we asked whether the vDNA would be recruited into a NISP multi-protein complex

403 using a modified chromatin immunoprecipitation (ChIP) assay (Fig 7). In our assay, proteins and

404 DNA were first cross-linked with formaldehyde and then the protein-DNA complexes were

405 immunoprecipitated with antibody (anti-GFP for NISP-GFP) from total protein extracts. The 
untransformed line (Col-0) and NISP-GFP-overexpressing (AtNISP-1, AtNISP-2, and AtNISP-3,

407 Fig 7a) lines were infected with CabLCV infectious clone and the modified ChIP assay was

408 performed using an anti-GFP antibody. We confirmed first that the inoculated plants were infected

409 (IN) by performing PCR from input DNA using CabLCV-specific primers, which amplify a 770-

410 bp fragment from CabLCV DNA-B (Fig 7b and S11b Fig, primers F1/R1) and a 389-bp fragment

411 from DNA-A (Fig 7e and S11a Fig, primers F1/R1). This viral DNA-B and DNA-A fragments

412 were also amplified from ChIP-DNA of infected (IN) AtNISP-overexpressing lines but not of

413 infected (IN) or uninfected (UN) Col-0, which lacks GFP-tagged NISP (Fig 7a, 7e). Using two

414 sets of DNA-B-specific primers (F2/R2 and F3/R3, S10b Fig), ChIP-qPCR further demonstrated

415 significant enrichment of DNA-B-specific fragments in infected AtNISP-GFP-overexpressing

416 samples, which had been precipitated with anti-GFP serum, but not in pulled down samples from

417 infected Col-0 lines (Fig 7c and 7d). Likewise, the use of F2/R2 set of DNA-A primers (S10a Fig

418 10a) also resulted in significant enrichment of the DNA-A-specific fragment in ChIPed DNA from

419 infected AtNISP-GFP-overexpressing samples but not from infected Col-0 (Fig 7f). Collectively,

420 our results indicate that NISP is a functional component of the vDNA-complex that traffics along

421 the cytoplasm to the cell periphery for the MP-mediated cell-to-cell movement of vDNA into

422 adjacent cells.

423

\section{Discussion}

Viruses are obligate intracellular parasites and, once in the host cell cytoplasm, they must

427 move intracellularly to and from the replication site to the plasma membrane for spreading. The 428 cytoplasmic limitations on free diffusion have forced viruses to evolve efficient mechanisms to 
429 manipulate the host transport system for their benefit. Understanding how viruses hijack the host

430 intracellular transport system using a limited repertoire of proteins provides an opportunity to

431 uncover the molecular bases of the host transport machinery. Bipartite begomoviruses, plant

432 ssDNA viruses, use two movement proteins, MP and NSP, to move intracellularly from the site of

433 replication in the nucleus to the cell surface [17]. However, the host components of the vDNA

434 complex competent for intracytoplasmic translocation have not been identified, and the underlying

435 mechanism for intracytoplasmic trafficking of vDNA remains to be elucidated. Here, we used a

436 previously fabricated, in situ synthesized protein microarray containing 4600 Arabidopsis ORFs

437 [37] to identify NSP- and MP-Arabidopsis protein-protein interactions (PPIs). Consistent with the

438 movement function of the viral proteins, the identified NSP-MP-PPI network uncovered direct and

439 indirect interactions, over-represented under transport activity, protein binding, membrane-bound

440 organelles, intracellular vesicle, and SNARE complex ontology terms, which may define an

441 intracellular route for vDNA trafficking. Precedents in the literature have implicated a

442 microtubule-associated intracellular movement of viral proteins and vDNA in addition to an

443 endocytic recycling pathway for the MP-mediated cell-to-cell movement of vDNA protein

444 complexes $(35 ; 36]$. Accordingly, transport function-related proteins, including kinesin motor

445 protein, plant syntaxin 121, Rab GTPase, Ras, Got1/Sft2-like vesicle transport protein, target

446 SNARE coiled-coil domain protein, syntaxin-6 plant proteins, prenylated Rab acceptor, Heat

447 shock protein 70 (Hsp 70), nuclear pore anchor, synaptotagmin A, were over-represented in the

448 MP-NSP- PPI network. These studies thus provided a critical framework for future investigations

449 to elucidate the molecular mechanisms for intracytoplasmic transport of begomoviruses.

450 The NSP-interacting syntaxin domain-containing protein (NISP) is identified as a new

\section{1 functional cellular partner of begomovirus NSP}


In the present investigation, we characterized an NSP-specifically interacting syntaxin-6

453 domain-containing protein, designated NISP and provided several lines of evidence indicating that

454 NISP might be involved in the intracytoplasmic movement of NSP-vDNA. First, co-localization

455 studies using organelle-associated molecular markers, and the FM4-64 marker demonstrated that

456 NISP is associated with TGN/early endosomal vesicles. Second, we confirmed that NISP

457 interacted with NSP in planta and showed that the complex formation occurred in vesicle-like

458 structures, which resemble the NISP-associated TGN/endosomal vesicles, the typical subcellular

459 localization of syntaxin-6-like proteins from Arabidopsis and humans [49]. Third, we showed that

460 NISP exhibits a pro-viral function, and begomovirus infection required NISP-NSP interaction. The

461 mutant nisp-1 was less susceptible nt to begomovirus as the knockout lines displayed attenuated

462 symptoms, a delayed course of infection, and accumulated much lower viral DNA as compared to

463 Col-0. In contrast, the overexpressing lines were more susceptible to begomovirus. We took

464 advantage of a highly conserved NISP paralog, AT2G18860, to show that NISP-NSP interaction

465 was the molecular basis for the NISP pro-viral function. AT2G18860, which shares with NISP a

$46678,6 \%$ identical syntaxin-6 domain, did not interact with NSP and thus did not interfere with

467 begomovirus infection. Consistent with these data, NISP, but not AT2G18860, is induced by

468 begomovirus infection. Fourth, NISP also interacted with NIG, which facilitates the release of the

469 NSP-DNA complex from the nuclear pores to the cytosol $[12,18]$, and the presence of NSP

470 enhanced the NISP-NIG complex formation, indicating that NSP, which interacts with NIG [12]

471 and NISP (Fig 4), may be recruited by the complex. We used ChIP assay to demonstrate that NISP

472 was also associated with vDNA in infected cells, which may be assembled into a NISP-NIG-NSP

473 multiprotein complex. Finally, the NISP interactions relocated the dispersed cytosolic NIG and

474 viral NSP to trafficking vesicle resembling the NISP-containing spherical structure associated with 
475 TGN/endosome. The NISP-mediated translocation of NSP-vDNA to endosomal vesicles favors

476 the association of the viral complex with MP, which is brought to the endosome via interaction

477 with the endosome-associated SYTA for the MP-mediated cell-to-cell movement of vDNA [35].

478

479 NISP belongs to a new, previously unidentified plant-specific subfamily of syntaxin-6-like 480 proteins

482 eukaryotic organisms divided this family into two subclades, one containing proteins from all 483 tested organisms, including SYP61 from Arabidopsis, and a new, previously unidentified plant484 specific subclade, which includes NISP and AT2G18860 paralogs clustered in pair. SYP61 has 485 been shown to form functional SNARE complexes involved in the vacuolar-TGN recycling 486 pathway and in exocytotic trafficking to the plasma membrane $[43,50]$. The process of membrane

487 fusion is the final step of vesicle transport, which is generally driven by a specific pairing of $1 \mathrm{v}-$ 488 SNAREs with the 2 or 3 cognate t-SNARE molecules into a trans-SNARE complex [49, 51]. The 489 proteins from the SYP61 subclade contain the typical structural configuration of t-SNARES, 490 including the N-terminal syntaxin-6 domain, followed by a C-terminal t-SNARE coiled-coil 491 homology domain and a transmembrane segment at the C-terminus. The proteins from the NISP 492 subclade harbor the N-terminal syntaxin-6 domain and the transmembrane segment but lack a C493 terminal SNARE domain, which may impair the participation of these new syntaxin-6-like proteins

494 in the assembly of a functional trans-SNARE complex for membrane fusion. Nevertheless, we 495 have demonstrated that a representative of this new syntaxin- 6 subclade, NISP, is functionally 496 expressed in plant cells. In addition to being induced by begomovirus infection, NISP functions as 497 a susceptibility factor to begomoviruses, forms complexes with viral and host proteins and is 
498 located in TGN/endosome-associated vesicles. We also mapped the NSP-binding domain on NISP

499 to the syntaxin-6 domain. Therefore, we propose that NISP may function as an intracellular vesicle

500 docking site to which the cargo proteins are specifically recruited through interactions with the

501 syntaxin-6-like domain.

We also showed that members of the new syntaxin-6 subclade might diverge functionally

503 concerning protein-protein binding specificity. The NISP paralog, AT218860, which shares with

504 NISP a conserved syntaxin-6 domain (78.6\% identity), fails to interact with viral NSP and hence

505 does not affect begomovirus infection. Therefore, specificity of protein interactions may rely on

506 marginal features of the syntaxin- 6 domain. A striking difference between NISP and AT2G18860

507 is an eight-amino acid deletion toward the N-terminus of the syntaxin-6 domain, which may

508 account for the NISP specificity to NSP (S4 Fig). Complementary studies are necessary to

509 precisely define the determinants of a functional syntaxin-6 domain for NSP interaction, which

510 will be useful for engineering resistance to begomoviruses.

\section{NISP may participate in the intracytoplasmic transport of NSP-vDNA}

513 Based on the current data and others previously reported data, we propose a model for the

514 NISP integration into an intracytoplasmic transport route used for the begomovirus anterograde

515 movement. Begomoviruses replicate in the nuclei of infected cells, where NSP interacts with

516 newly-synthesized vDNA and facilitates the translocation of vDNA to the cytoplasm via nuclear

517 pores $[13,32])$. This nuclear exportation process may be mediated by an exportin-like protein yet

518 to be identified. At the cytosolic side of the nuclear envelope, NIG associates with the NSP-vDNA

519 complex to accessorize the release of NSP-vDNA from the nuclear pores into the cytosol [12,18].

520 The pro-viral function of NIG is associated with its cytosolic localization because the WWP1- 
521 mediated recruitment of NIG into nuclear bodies impairs begomovirus infection [48]. At the

522 cytosol, we showed here that NISP may recruit the NIG-NSP-vDNA complex to TGN/endosome-

523 associated transport vesicles. The nature of the NISP-associated vesicle was identified using cell

524 biology molecular markers and FM4-64, which demonstrated that NISP is predominantly localized

525 at TGN and in the early endosome (Fig 3), which was consistent with the localization of syntaxin-

5266 proteins in most eukaryotic cells [40]. As MP has been demonstrated to associate with the

527 endosomal SYTA, a possible NISP sequestration of NSP-DNA into TGN/endosome-associated

528 vesicles may provide the means for the predicted interactions between endosomally localized MP-

529 SYTA and NSP-DNA, which would favor the MP-mediated intercellular transport of vDNA via

530 an endocytic recycling pathway [35]. In summary, our data support a model in which direct

531 interactions between the host factor NISP and NIG/NSP modulate vDNA intracellular trafficking

532 to endosomes where MP, recruited by the endosomal SYTA, opportunistically interacts with NSP-

533 vDNA to mediate the cell-to-cell movement of vDNA via plasmodesmata.

\section{Material and Methods}

\section{Phylogenetic analysis}

538 The amino acid full-length sequences of plant proteins were retrieved from TAIR

539 (http://arabidopsis.org/), Phytozome v12 database (https://phytozome.jgi.doe.gov/) and amino acid

540 full-length sequences of other species from the Ensemble database

541 (https://www.ensembl.org/index.html). The syntaxin domain superfamily was predicted using the

542 software HMMER v3.2.1 (http://hmmer.org/) using Pfam v.30 database (https://pfam.xfam.org/).

543 The syntaxin domain-containing protein sequences were selected to perform the phylogenetic 
544 reconstruction. The amino acid sequences were aligned using the MUSCLE algorithm [52].

545 Phylogenetic trees were constructed using Bayesian Inference with MrBayes v3.2.2 [53] and mix

546 model of amino acid substitution (Wag). The analyses were performed running 10.000.000

547 generations and excluding the first 2.500.000 generations as burn-in and visualized with Figtree

548 software (http://tree.bio.ed.ac.uk/software/figtree/). Plant NISP homologous proteins were

549 identified using the BLAST algorithm implemented in the Ensemble and Phytozome databases.

550 The amino acid sequences of the NISP homologs were selected for phylogenetic reconstruction of

551 N-terminal Syntaxin-6 family using the same tools and criteria applied for the superfamily

552 syntaxin. However, the amino acid substitution model Jones was used for the N-terminal Syntaxin-

5536 phylogenetic tree.

554

555 PPI network analysis

556 NSP- and MP-interacting proteins from Arabidopsis were used as a query term to identify

557 their respective interactions described in the BAR database (Bio-Analytic Resource for Plant

558 Biology, http://bar.utoronto.ca/interactions/). The Biogrid and IntAct databases were selected for

559 searching. The protein-protein interactions (PPI) were retrieved and imported into the Cytoscape

560 software (https://cytoscape.org/) to visualize the topology of the PPI network and to calculate the

561 network centrality metrics for each protein. The measured network centralities were betweenness,

562 closeness, eccentricity, and degree. Briefly, the betweenness centrality in the PPI network of the

563 graph $\mathrm{G}=(\mathrm{V}, \mathrm{E})$ was calculated by observing the number of times a node $\mathrm{v}$ (protein) contributes

564 as a link along the shorter paths among all nodes. The betweenness centrality of this node can be

565 analyzed as:

$$
\sum_{s, t \in V} \frac{\sigma(s, t \mid v)}{\sigma(s, t)}
$$


567 where $\mathrm{V}$ is the total set of nodes, $\sigma(s, t)$ is the number of shorter paths of $(s, t)$, and $\sigma(s, t \mid v)$ is the

568 number of paths crossing $v[54]$. The betweenness centrality can indicate the relevance of a protein

569 as functionally capable of keeping together interacting proteins. Closeness centrality (of a node v

570 is the sum of the shortest path distances from $w$ to all other nodes and calculated as described by

571 Freeman [55]:

$$
\frac{1}{\sum_{w \in V} \operatorname{dist}(v, w)}
$$

575 A high closeness value can be determined by the fact that all nodes are usually close to node v.

576 The eccentricity centrality of a node $\mathrm{v}$ is the maximum distance from $\mathrm{v}$ to all other nodes in graph

$577 \mathrm{G}$ and can be analyzed as described by Hage and Harary [56]:

578

$$
\frac{1}{\max \{\operatorname{dist}(v, w): w \in V\}}
$$

581 In addition, the degree centrality of node $v$ was calculated by $\sum i=1 K_{d i}$, where $d$ represents each

582 adjacent node and $K$, the total number of adjacent nodes. Nodes with a high degree can be 583 designated hubs and hold together different nodes with a lower degree.

\section{GO analysis of the PPI network}

585 The gene set enrichment analysis (GSEA) of the proteins that compose the PPI network 586 was performed using the Bioconductor GOstats package [57]. GSEA analysis computes 587 hypergeometric test $\mathrm{p}$ values for over- or underrepresentation for each GO term, and the annotation 588 of genes is based on the Bioconductor annotation package org.At.tair. 


\section{Plasmid constructions}

All recombinant plasmids were generated by the GATEWAY cloning system (Invitrogen, http://www.invitrogen.com/). The primers used for gene amplification are listed in S3 Table. The coding regions of NISP (AT4G30240) and AT2G18860, with or without a translational stop codon,

594 were amplified from leaf cDNA of Arabidopsis thaliana ecotype Columbia (Col-0) and inserted 595 into the entry vectors pDONR201 and pDONR207. The resulting clones were designated 596 At4g30240-St-pDONR201 (pUFV2134), At4g30240-Ns-pDONR201 (pUFV2135), At2g18860597 St-pDONR201 (pUFV3193), At2g18860-Ns-pDONR201 (pUFV3194), At4g30240-St598 pDONR207 (pUFV2236), At4g30240-Ns-pDONR207 (pUFV2237), At2g18860-St- pDONR207 599 (pUFV3195), At2g18860-Ns-pDONR207 (pUFV3196). Then, the DNA inserts were transferred 600 by recombination from the entry vector to different yeast and plant expression vectors.

601 For the yeast two-hybrid assay, the NISP and AT2G18860 coding regions were transferred 602 from the respective entry vectors (At4g30240-St-pDONR201 and At2g18860-St-pDONR201) to 603 the yeast expression vectors pDEST32 and pDEST22, generated the clones At4g30240-St604 pDEST32 (pUFV2141), At4g30240-St-pDEST22 (pUFV2142), At2g18860-St-pDEST32 605 (pUFV3197) and At2g18860-St-pDEST22 (pUFV3198), fused to binding domain (BD) 606 (pDEST32) or activation domain (AD) (pDEST22) of GAL4. The NSP-and NIG-derived clones 607 used in the yeast two-hybrid assay have been previously described and designated NSP-St608 pDEST32, NSP-Cl-pDEST22 (Fontes et al., 2004), and NIG-pDEST22 (pUFV2547; Calil et al., 609 2018).

610 For the subcellular localization experiments, the NISP and AT2G18860 coding region were 611 fused to GFP and expressed in planta under the control of the CaMV 35S promoter in the vector 
612 pK7FWG2. For the co-immunoprecipitation assay, in addition to the GFP fusion, NISP ORF was

613 also fused to hemagglutinin (HA) tag, using the binary destination vector $\mathrm{pK} 7 \mathrm{~m} 34 \mathrm{GW}$ for three

614 fragment recombination. The resulting DNA constructions were At4g30240-pK7FWG2

615 (pUFV2576), At2g18860-pK7FWG2 (pUFV3199) and 2x35S::At4g30240-Ns-6HA-pH7m34GW

616 (pUFV2586).

617 For the subcellular co-localization assays, the ER-associated molecular marker ER-

618 mCherry (CD-959), the TGN-associated molecular marker G-mCherry (CD-967) and the

619 tonoplast-associated molecular marker vac-mCherry (CD-975) were obtained from the

620 Arabidopsis Biological Resource Center. The plasma membrane-associated molecular marker,

621 FLS2-mCherry was obtained by amplifying the FLS2 ORF from Arabidopsis cDNA with the

622 primers AtFLS2-Fwd and AtFLS2-NS-Rvs (S3 Table), inserting the amplified fragment by

623 recombination into pDON207 and transferring it by triple recombination to the destination vector

$624 \mathrm{pH7m34GW}$ along with FLS2-Ns-pDON207, pDONR-P4-P1R+2x35S, and pDONR-P2R-

625 P3+mCherry, resulting in the clone FLS2-mCherry (pUFV3034). Likewise, the SYTA ORF was

626 amplified from leaf cDNA with the primers AtSYTA-Fwd and AtSYTA-NS-Rvs (S3 Table),

627 cloned into the entry vector pDONR-207 and transferred via triple recombination to the destination

628 vector $\mathrm{pH} 7 \mathrm{~m} 34 \mathrm{GW}$ along with SYTA-NspDON207, pDONR-P4-P1R+2x35S, and pDONR-P2R-

629 P3+mCherry, generating SYTA-mCherry (pUFV3296).

630 The NISP coding region was also fused to GFP and expressed in plants under the control

631 of its endogenous promoter. For this, about $2000 \mathrm{bp}$ of 5' flanking sequence of At4g30240 was

632 PCR-amplified using the primers described in S3 Table and cloned into pDONR-P4-P1R, yielding

633 proAt4g30240-pDONR-P4-P1R (pUFV2436). Then, the 5' flanking sequence was transferred by

634 triple recombination to the destination vector pK7Fm34GW along with At4g30240-Ns-pDON201, 
635 proAt4g30240-pDONR-P4-P1R, and eGFP-pDONR-P2R-P3, resulting in the clone 636 proAt4g30240::At4g30240-GFP (pUFV2849). The NSP- and NIG-derived clones used for co637 immunoprecipitation assays have been previously described and designated as $2 \times 35 \mathrm{~S}-\mathrm{NSP}-\mathrm{Cl}-$ 638 6HA-pH7m34GW (pUFV2424), NSP-Cl-pK7FWG2(pUFV2536), 2x35S-NIG-Ns-6HA639 pH7m34GW (pUFV1946) and NIG- pK7FWG2 (pUFV1177) [48].

640 The SPYNE-GW and SPYCE-GW vectors, which contain the N-terminal (nYFP) and C641 terminal (cYFP) of YFP, respectively, were used for the BiFC experiments. At4g30240-Ns642 pDONR207 was transferred to SPYNE-GW and SPYCE-GW, yielding At4g30240-Ns-SPYNE 643 (pUFV2238) and At4g30240-Ns-SPYCE (pUFV2239). The NSP- and NIG-derived clones used in 644 the BiFC assays have been previously described and designated as NSP-Cl-Ns-SPYNE 645 (pUFV1654), NSP-Cl-Ns-SPYCE (pUFV1655), NIG-Ns-SPYNE (pUFV1646), and NIG-Ns646 SPYCE (pUFV1647) [48].

647 NISP truncated versions were obtained from At4g30240-St-pDONR201 by PCR using 648 primers listed in S3 Table. The generated fragments were cloned into the entry vector pDONR201 649 and designated as At4g30240(1-107)-St-pDONR201 (pUFV2806), At4g30240(105-200)-St650 pDONR201 (pUFV2807) and At4g30240(200-300)-St-pDONR201 (pUFV2808). The respective 651 DNA inserts were then transferred to the yeast expression vectors, yielding At4g30240(1-107)-St652 pDEST32 (pUFV2822), At4g30240(1-107)-St-pDEST22 (pUFV2819), At4g30240(105-200)-St653 pDEST32 (pUFV2823), At4g30240(105-200)-St-pDEST22 (pUFV2820), At4g30240(200-200)654 St-pDEST32 (pUFV2824) and At4g30240(200-300)-St-pDEST22 (pUFV2821). 655 656 Detecting MP- and NSP-host protein interactions on high-density halo tag-NAPPAs 
The identification of NSP and MP partners was carried out through a previously fabricated by Yazaki et al. [37]. The protein microarray, which consisted of a set of in situ synthesized

660 proteins from 4600 Arabidopsis thaliana ORFs, has been designated AtNAPPA01. $3 \times$ HA-fused

661 CabLCV MP and NSP proteins were independently expressed using the TNT system (Promega)

662 according to the manufacturer's recommendations. The in vitro synthesized HA-fused MP, and

663 HA-fused NSP were used to probe individually AtNAPPA01. The identification of MP- and NSP-

664 interacting proteins with the NAPPA protein-protein interaction method was from a single 665 experiment using duplicated spots on one glass slide. Interactions were considered positive only 666 when both replicated spots displayed normalized signal intensity above background. Experiments, 667 data scanning, quantification, and data processing were carried out as described by Yazaki et al. $668[37]$.

\section{Plant growth conditions and transformation}

Arabidopsis thaliana, ecotype Columbia (Col-0), was used as the wild type. Homozygous

672 seeds from the T-DNA insertional mutants nisp-1 (Salk_117751) and at2g18860-1 (Salk_099699)

673 were obtained from the Arabidopsis Biological Resource Center. Arabidopsis seeds were surface-

674 sterilized and cold treated at $4^{\circ} \mathrm{C}$ for $72 \mathrm{~h}$ in the dark and then exposed to white light. Seedlings

675 were grown at $22^{\circ} \mathrm{C}$ on plates containing Murashige-Skoog (MS) medium for 2 weeks and then

676 transferred to soil. Plants were grown in a growth chamber at $22{ }^{\circ} \mathrm{C}$ under short-day conditions

677 (12h light/12h dark). Transgenic lines were obtained via A. tumefaciens-mediated transformation

678 using the floral dip method [58]. Transformed plants with the indicated DNA constructions were

679 selected in MS medium supplemented with the appropriate antibiotic (kanamycin, pK7FWG2). 
680 Selected transformants were confirmed by PCR, transferred to soil, and grown in a growth

681 chamber at $22^{\circ} \mathrm{C}$ under long-day conditions (16h light/8h dark) to generate seeds. For segregation

682 analysis, seeds were germinated on MS medium containing $50 \mu \mathrm{gmL}^{-1}$ kanamycin. Homozygous

683 T1 lines for the T-DNA loci were selected by determining the frequency of their kanamycin-

684 resistant T2 seeds after self-pollination. Accumulation of NISP-GFP and AT2g18860-GFP

685 transcript and protein levels were monitored in each generation by qRT-PCR using gene-specific

686 primers (S3 Table) and immunoblotting using anti-GFP antibody. From the independently

687 transformed lines (8-15 lines of each construct and genotype transformed), we selected three 688 independently transformed lines which appeared to have an integrated T-DNA locus on a single 689 chromosome, since $75 \%$ of their T1 segregating seedlings were resistant to kanamycin (S4 Table).

690 The selected lines are presented in S4 Table. The nomenclature nisp-1/NISP-1-3 indicates that the 691 genotype nisp-1 was transformed with At4g30240-pK7FWG2, harboring the NISP ORF fused to

692 the N-terminus of GFP under the control of the 35S promoter to generate NISP-complementing 693 lines. The designations AtNISP-1, AtNISP-2 and AtNISP-3 indicate three independently 694 transformed lines, which were generated by transformation of Col-0 with At4g30240-pK7FWG2 695 to obtain NISP-GFP-overexpressing lines. The same nomenclature was applied for the 696 transformation of at2g18860-1 mutant and Col-0 with At2g18860-pK7FWG2, containing the 697 At2g18860 ORF fused to the N-terminus of GFP under the control of 35S promoter (S4 Table).

699 RNA isolation and qRT-PCR analysis 
reverse transcribed using oligo $(\mathrm{dT})(0,5 \mu \mathrm{M})$ and $1 \mathrm{U}$ of M-MLV (Thermo). Real-time RT-PCR

705 primers in $10 \mu \mathrm{L}$ of reaction and an ABI7500 real-time PCR system (Life Technologies). The

706 expression of each gene was normalized to the expression of Actin and quantified using the $2^{-\Delta \mathrm{Ct}}$

707 method. The primers used to detect specific transcripts with real-time RT-PCR are listed in S3

708 Table.

709

710 Yeast two-hybrid assay

711 Competent cells of Saccharomyces cerevisiae, strain AH109 (MATa, trp1-901, leu2-3,

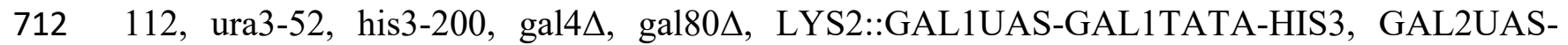

713 GAL2TATA-ADE2, URA3::MEL1UAS-MEL1 TATA-lacZ), were cotransformed with the yeast

714 expression vectors pDEST22 (Gal4 AD) and pDEST32 (Gal4 BD), carrying the coding region of

715 the tested proteins, along with $100 \mu \mathrm{g}$ of salmon sperm carrier DNA (ssDNA), using the lithium

716 acetate/polyethylene glycol (PEG) method. For the selection of double transformants, the cells

717 were plated on medium deficient in leucine and tryptophan (SD, Synthetic Dropout, -Leu, -Trp)

718 and cultured for 4 days at $28^{\circ} \mathrm{C}$. Interactions were monitored by the ability of the reporter strain to

719 grow on media lacking leucine, tryptophan, and histidine but supplemented with 2.5 mM 3-AT (3-

720 Amino-1,2,4-triazole; Sigma) for 4 days at $28^{\circ} \mathrm{C}$.

721

\section{Co-immunoprecipitation assay}

723 The in vivo interactions between NISP and NSP or NIG were monitored by co-

724 immunoprecipitation using the $\mu \mathrm{MACS}^{\mathrm{TM}}$ Epitope Tag Protein Isolation (MACS/Miltenyil Biotec)

725 kit, according to manufacturer's recommendations. Agrobacterium tumefaciens (strain GV3101)- 
726 mediated transient expression in N. benthamiana leaves was carried out as described (12). Total

727 protein extracts were obtained from infiltrated leaves expressing the recombinant proteins. At 48

$728 \mathrm{~h}$ after agro-infiltration, $200 \mathrm{mg}$ of leaves were homogenized with $1 \mathrm{ml}$ of lysis buffer $(50 \mathrm{mM}$

729 Tris- $\mathrm{HCl} \mathrm{pH}$ 8.0, $1 \mathrm{mM}$ PMSF, $2 \mathrm{mM}$ benzamidine, 1\% (v/v) Nonidet P-40) and incubated for $2 \mathrm{~h}$

730 with anti-green fluorescent protein (GFP) magnetic beads (MACS / Miltenyi Biotec) at $4{ }^{\circ} \mathrm{C}$ under

731 rotation. After incubation, the extracts were applied to a MACS column for the indicated protein

732 tag, washed five times before elution with a pre-heated buffer at $95{ }^{\circ} \mathrm{C}$. The immunoprecipitated

733 proteins were separated by sodium dodecyl sulfate- polyacrylamide gel electrophoresis (SDS-

734 PAGE) $10 \%(\mathrm{p} / \mathrm{v})$, immunoblotted with the monoclonal antibodies anti-human influenza

735 hemagglutinin (anti-HA) epitope tag, amino acids 98-106 (Miltenyi Biotec, 130-091-972;

736 1:10.000) or anti-GFP (Miltenyi Biotec, 130-091-833; 1:10.000), and detected using Signal West

737 Pico Chemiluminescent Substrate (Thermo Scientific) according to the recommendations of the

738 manufacturer.

739

\section{Bimolecular fluorescence complementation (BiFC) assay}

741 Different combinations of A. tumefaciens strain GV3101 expressing the indicated proteins

742 fused to N-YFP/C-YFP were co-infiltrated into the abaxial surface of $N$. benthamiana leaves at

$743 \mathrm{OD}_{600 \mathrm{~nm}}$ of 1:1 proportion. Three days after infiltration, fluorescence was examined in epidermal

744 cells using a Zeiss inverted 510 META laser scanning microscope equipped with an argon laser

745 and a helium laser as excitation sources. YFP was excited at $514 \mathrm{~nm}$ using the argon laser, and

746 YFP emission was detected using a 560-615-nm filter. The images were processed using LSM

747 Image Browser 4 (ZEISS) software. 


\section{Subcellular localization}

751 leaves transiently expressing GFP fusions. Briefly, $N$. benthamiana leaves were agro-infiltrated

752 with $A$. tumefaciens strain GV3101 carrying proAt4g30240-pDONR-P4-P1R (pUFV2436),

753 At4g30240-pK7FWG2 (pUFV2576) or At2g18860-pK7FWG2 (pUFV3199) (Method S1) and

754 fluorescence of the GFP fusions were visualized in epidermal cells 3 days after infiltration by

755 confocal microscopy using a Zeiss inverted 510 META laser scanning microscope equipped with

756 an argon laser and a helium laser as excitation sources. GFP was excited by the argon/helium-neon

757 laser system in $488 \mathrm{~nm}$ wavelength, and emission was collected in 500-530 nm band-pass filter.

758 Images were captured and processed with LSM Image Browser 4 (Carl-Zeiss) software.

759 For co-localization assays, NISP-GFP was co-expressed with ER-, Golgi-, tonoplast-,

760 plasma membrane- and endosome-associated molecular markers fused to mCherry (see in plasmid

761 constructions) and fluorescence was examined by confocal microscopy, using a Zeiss inverted

762 LSM510 META laser scanning microscope, on whose system, the default scanning mode is

763 sequential scanning (multitrack mode). For imaging GFP, the $488 \mathrm{~nm}$ excitation line and the 500

764 to $530 \mathrm{~nm}$ band pass filter were used. Excitation of mCherry was at $540 \mathrm{~nm}$ and emission of 608-

$765680 \mathrm{~nm}$. For the FM4-64 staining, $48 \mathrm{~h}$ after agro-infiltration with the DNA construct expressing

766 NISP-GFP, the leaves were infiltrated with a solution of $50 \mu \mathrm{M}$ FM4-64 (Molecular Probes,

767 Eugene, OR) and examined by confocal microscopy $1 \mathrm{~h}$ post-infiltration.

768

769 Infectivity assay

770 The generation of transgenic lines overexpressing NISP (AtNISp) and At2g18860

771 (AT2G18860), knockout lines (nisp-1 and at2g18860-1), NISP complementing lines are described 
772 in Method S1. Transgenic lines and Columbia at the seven-leaf stage were inoculated with

773 plasmids containing partial tandem repeats of CabLCV DNA-A and DNA-B by biolistic delivery

774 using a microprojectile bombardment model PDS-1000/He accelerator at 900 psi. $(24,26,59)$. The

775 inoculated plants were transferred to a growth chamber and monitored for symptoms development

776 and progress of infection [23, 60]. Total DNA was extracted from systemically infected leaves

777 [61], and viral DNA accumulation was detected by PCR using universal DNA-B specific primers

778 PBL1v2040 and PCRC1 [62] (S3 Table). In each experiment, 15 plants of each line were

779 inoculated with $2 \mu \mathrm{g}$ of tandemly repeated DNA-A plus DNA-B per plant. The course of infection

780 was examined using data from three independent experiments.

781

782 Quantification of viral DNA in infected plants

783 The accumulation of viral DNA in infected plants was quantified by qPCR [26]. The

784 reactions were prepared in $10 \mu \mathrm{L}$ using SYBR Green Master Mix (Life Technologies), $100 \mathrm{ng}$ of

785 total DNA from systemically infected leaves, and CabLCV-DNA-B-specific primers (S3 Table),

786 and analyzed on a real-time PCR system. The genomic copies of CabLCV were normalized against

787 an internal control (18S rRNA) to account for template input variation between tubes. For viral

788 DNA quantification, standard curves were prepared using serial dilutions of DNA-B of CabLCV

789 (100 to $10^{6}$ copies of viral genome per reaction).

790

791 Formaldehyde fixation and immunoprecipitation of NISP-GFP:vDNA complexes

792 A modified ChIP assay evaluated the in vivo interaction between NISP and viral DNA.

793 NISP-overexpressing transgenic lines (AtNIS) and Columbia (Col-0) at the six-leaf stage were

794 inoculated by biolistic delivery with CabLCV DNA-A and DNA-B infectious clones. Tissue 
795 harvest, formaldehyde fixation, and chromatin breaks from 45 days-old infected plants were 796 conducted according to Alves et al. [63] and Yamaguchi et al. [64]. For cross-linking proteins to 797 DNA with formaldehyde, $600 \mathrm{mg}$ of infected leaves were washed with $10 \mathrm{~mL}$ of PBS (10 mM $798 \mathrm{Na} 2 \mathrm{HPO} 4,1.8 \mathrm{~mm} \mathrm{KH} 2 \mathrm{PO} 4,137 \mathrm{mM} \mathrm{NaCl}, 2.7 \mathrm{mM} \mathrm{KCl}, \mathrm{pH}$ 7.4) and fixed in $10 \mathrm{~mL}$ PBS by 799 adding formaldehyde to $1 \%$ final concentration. The infiltration of formaldehyde into vegetable 800 cells was carried out under vacuum at $-500 \mathrm{~Pa}$ for $1.5 \mathrm{~min}$, then $1 \mathrm{~min}$ twice with $30 \mathrm{~s}$ intervals. 801 After washing first with $0.125 \mathrm{M}$ glycine under vacuum for $1.5 \mathrm{~min}$ and then with PBS, the tissue 802 was air-dried. The fixed samples were homogenized in liquid N2 and $1 \mathrm{~mL}$ of extraction buffer 803 (50 mM Tris-HCl pH 8.0, 10 mM EDTA, 1\% (v/v) Nonidet-P40). After incubation for $30 \mathrm{~min}$ on 804 ice, cell debris was removed by centrifugation at $12000 \mathrm{x}$ g for $10 \mathrm{~min}$. The supernatant $(300 \mu \mathrm{L})$ 805 was diluted with $700 \mu \mathrm{L}$ of the ChIP dilution buffer $(16.7 \mathrm{mM}$ Tris- $\mathrm{HCl} \mathrm{pH}$ 8,0, $167 \mathrm{mM} \mathrm{NaCl}$, $1.2 \mathrm{mM}$ EDTA, $0.01 \% \mathrm{SDS}$ ) and DNA was fragmented by sonication at an $80 \mu \mathrm{m}$ amplitude level, 807 six pulses for $30 \mathrm{~min}, 30 \mathrm{~s}$ on/off on the ice. Then, $700 \mu \mathrm{L}$ of the sonicated suspension was mixed 808 with $200 \mu \mathrm{L}$ of the ChIP dilution buffer, supplemented with $1.1 \%(\mathrm{v} / \mathrm{v})$ Triton X-100 and 809 centrifuged at $13200 \mathrm{xg}$ for $5 \mathrm{~min}$ at $4^{\circ} \mathrm{C}$. The supernatant was incubated with $50 \mu \mathrm{L}$ of protein810 A-garose beads for $2 \mathrm{~h}$. After centrifugation at $900 \mathrm{x} \mathrm{g}$ for $3 \mathrm{~min}, 18 \mu \mathrm{L}$ of the supernatant were 811 stored at $-80^{\circ} \mathrm{C}$, as input control. The remaining supernatant was incubated with $1 \mu$ of polyclonal 812 anti-GFP serum (Life Technologies A11122; 3x diluted) for $4 \mathrm{~h}$ at $4^{\circ} \mathrm{C}$. Anti-GFP-NISP813 GFP:vDNA complexes were captured by incubation with $50 \mu \mathrm{L}$ of potein-A-garose beads for $4 \mathrm{~h}$ 814 at $4^{\circ} \mathrm{C}$, under gentle agitation. After centrifugation at $900 \mathrm{x}$ g for $3 \mathrm{~min}$, the pellet was washed five 815 times with different washing buffers in the order: twice with the washing buffer A $(0.1 \%(w / v)$ 816 SDS 1\% (v/v) Triton X-100, 2 mM EDTA, 20 mM Tris-HCl, pH 8,1, $150 \mathrm{mM} \mathrm{NaCl),} \mathrm{once} \mathrm{with}$ 817 the buffer B (0.1\% (w/v) SDS 1\% (v/v) Triton X-100, 2 mM EDTA, $20 \mathrm{mM}$ Tris-HCl, pH 8,1, 
$818500 \mathrm{mM} \mathrm{NaCl})$, followed by the buffer C (250 mM LiCl, 1\% (v/v) IGEPAL-CA630, 1\% (w/v)

819 sodium deoxycholate, $1 \mathrm{mM}$ EDTA, $10 \mathrm{mM}$ Tris-HCl pH 8,1) and finally with 0,5 X TE (10 mM

820 Tris- $\mathrm{HCl} \mathrm{pH}$ 8,0, 1 M EDTA). The DNA pellet was reverse cross-linked from proteins and purified

821 using the QIAprep ${ }^{\circledR}$ Spin Miniprep kit.

822

823 ChIP-qPCR

824

NSP primers (S3 Table) were initially used to amplify viral DNA-B from the

825 immunoprecipitated DNA and input DNA to ensure DNA-B-specific amplification in samples.

826 Likewise, DNA-A-specific primers were used to examine initial amplification of the DNA-A

827 component. Each reaction contained $1 \mu \mathrm{L}$ of immunoprecipitated DNA or 20 -fold diluted input

828 DNA, $0.2 \mathrm{mM}$ CabLCV-DNA-B-specific primers (S3 Table), and $5 \mu \mathrm{L}$ of SYBR Green PCR

829 Master Mix (Life Technologies) in $10 \mu \mathrm{L}$ total volume. The thermal cycling conditions of the

830 reactions consisted of an initial step at $95^{\circ} \mathrm{C}$ for $10 \mathrm{~min}$, followed by 40 cycles at $94^{\circ} \mathrm{C}$ for $15 \mathrm{~s}$

831 and $58^{\circ} \mathrm{C}$ for $45 \mathrm{~s}$. The cycle threshold $(\mathrm{Ct})$ values from Col-0 and AtNISP were normalized with

832 the $\mathrm{Ct}$ values of the respective inputs, and the results were expressed as a percentage of the input.

833

\section{Author Contribution}

835 B.C.G.M performed most of the experiments; L.G.C.M. performed the co-localization

836 experiments and protein localization assays; M.D.-B., A.Y.K. and J.Y. performed protein

837 microarray assay; B.C.G.M. and J.P.B.M. performed two hybrid assays; J.C.F.S. performed in

838 silico and statistical analyses; B.C.G.M. and A.A.S. performed BiFC assays; E.P.B.F. and J.R.E.

839 conceived experiments; B.C.G.M. and E.P.B.F. designed all experiments, analyzed the data and

840 wrote the manuscript. 
842 Acknowledgments

843 The authors are grateful to the microscopy facility at Universidade Federal de Viçosa.

845 Funding

846 We are grateful to the Brazilian funding agencies CAPES, CNPq, FAPEMIG, and the National

847 Institute of Science and Technology in Plant-Pest interactions for supporting this study.

848

849 Competing interests

850

851 The authors have declared that no competing interests exist.

852

\section{References}

854 1. Dodding MP, Way M. Coupling viruses to dynein and kinesin-1. EMBO J. 2011;30: 3527-3539.

855 2. Schoelz JE, Harries PA, Nelson RS. Intracellular transport of plant viruses: finding the door out 856 of the cell. Mol Plant. 2011;4: 813-831.

857 3. Harries PA, Schoelz JE, Nelson RS. Intracellular Transport of Viruses and Their Components:

858 Utilizing the Cytoskeleton and Membrane Highways. Mol Plant-Microbe Interac. 2010;23: 13818591393.

860 4. Wolf S, Deom CM, Beachy RN, Lucas WJ. Movement protein of tobacco mosaic virus modifies 861 plasmodesmatal size exclusion limit. Science. 1989;246: 377-379.

862 5. Arriagada G. Retroviruses and microtubule-associated motor proteins. Cell Microbiol. 2017;19: 863 e12759.

864 6. Rojas MR, Macedo MA, Maliano MR, Soto-Aguilar M, Souza JO, Briddon RW, et al. World 865 Management of Geminiviruses. Ann Rev Phytopathol. 2018;56: 637-677. 
866 7. Hanley-Bowdoin L, Bejarano ER, Robertson D, Mansoor S. Geminiviruses: masters at

867 redirecting and reprogramming plant processes. Nat Ver Microbiol. 2013;11: 777-788.

868 8. Silva JCF, Carvalho TFM, Basso MF, Deguchi M, Pereira WA, Sobrinho RR, et al. Geminivirus

869 data warehouse: a database enriched with machine learning approaches. BMC Bioinformatics.

$870 \quad 2017 ; 18: 240$.

871 9. Pascal E, Sanderfoot AA, Ward BM, Medville R, Turgeon R, Lazarowitz SG. The geminivirus

872 BR1 movement protein binds single-stranded DNA and localizes to the cell nucleus. Plant Cell.

873 1994;6: 995-1006.

874 10. Rojas MR, Noueiry AO, Lucas WJ, Gilbertson RL. Bean dwarf mosaic geminivirus movement

875 proteins recognize DNA in a form- and size-specific manner. Cell. 1998;95: 105-113.

876 11. Hehnle S, Wege C, Jeske H. Interaction of DNA with the movement proteins of geminiviruses

877 revisited. J Virol. 2004;78: 7698-7706.

878 12. Carvalho CM, Fontenelle MR, Florentino, LH, Santos AA, Zerbini FM, Fontes EPB. A novel 879 nucleocytoplasmic traffic GTPase identified as a functional target of the bipartite geminivirus 880 nuclear shuttle protein. Plant J. 2008a;5: 869-880.

881 13. Sanderfoot AA, Lazarowitz SG. Cooperation in viral movement: The geminivirus BL1

882 movement protein interacts with BR1 and redirects it from the nucleus to the cell periphery. Plant 883 Cell. 1995;7: 1185-1194.

884 14. Noueiry AO, Lucas WJ, Gilbertson RL. Two proteins of a plant DNA virus coordinate nuclear 885 and plasmodesmatal transport. Cell. 1994;76: 925-932.

886 15. Lazarowitz SG, Beachy RN. Viral movement proteins as probes for intracellular and 887 intercellular trafficking in plants. Plant Cell. 1999;11: 535-548. 
888 16. Ward BM, Lazarowitz SG. Nuclear export in plants: use of geminivirus movement proteins 889 for a cell-based export assay. Plant Cell. 1999;11: 1267-1276.

890 17. Gafni Y, Epel BL. The role of host and viral proteins in intra and inter-cellular trafficking of 891 geminiviruses. Physiol Mol Plant Pathol. 2002;60: 231-241.

892 18. Carvalho CM, Machado JP, Zerbini FM, Fontes EP. NSP-interacting GTPase: a cytosolic 893 protein as cofactor for nuclear shuttle proteins. Plant Signal Behav. 2008b;3: 752-754.

894 19. Sanchez-Velar N, Udofia EB, Yu Z, Zapp ML. hRIP, a cellular cofactor for Rev function, 895 promotes release of HIV RNAs from the perinuclear region. Genes Dev. 2004;18:23-34.

896 20. Zhou Y, Rojas MR, Park M-R, Seo YS, Lucas WJ, Gilbertson RL. Histone H3 interacts and 897 colocalizes with the nuclear shuttle protein and the movement protein of a geminivirus. J Virol. $898 \quad 2011 ; 85: 11821-11832$.

899 21. McGarry RC, Barron YD, Carvalho MF, Hill JE, Gold D, Cheung E, et al. A novel Arabidopsis 900 acetyltransferase interacts with the geminivirus movement protein NSP. Plant Cell. 2003;15: 16059011618.

902 22. Carvalho MF, Lazarowitz SG. Interaction of the movement protein NSP and the Arabidopsis 903 acetyltransferase AtNSI is necessary infection and pathogenicity. J Virol. 2004;78: 11161-11171.

904 23. Florentino LH, Santos AA, Fontenelle MR, Pinheiro GL, Zerbini FM, Baracat-Pereira MC, et 905 al. A PERK-Like receptor kinase interacts with the geminivirus nuclear shuttle protein and 906 potentiates viral infection. J Virol. 2006;80: 6648-6656.

907 24. Fontes EPB, Santos AA, Luz DF, Waclawovsky AJ, Chory J. The geminivirus nuclear shuttle 908 protein is a virulence factor that suppresses transmembrane receptor kinase activity. Genes Dev. $909 \quad 2004 ; 18: 2545-2556$. 
910 25. Carvalho CM, Santos AA, Pires SR, Rocha CS, Saraiva DI, Machado JPB, et al. Regulated

911 nuclear trafficking of rpL10A mediated by NIK1 represents a defense strategy of plant cells against

912 virus. PLoS Pathog. 2008c;4: e1000247.

913 26. Zorzatto C, Machado JPB, Lopes KVG, Nascimento KJT, Pereira WA, Brustolini OJB, et al.

914 NIK1-mediated translation suppression functions as a plant antiviral immunity mechanism.

915 Nature. 2015;520: 679-682.

916 27. Sanderfoot AA, Ingham DJ, Lazarowitz SG. A viral movement protein as a nuclear shuttle.

917 The geminivirus BR1 movement protein contains domains essential for interaction with BL1 and 918 nuclear localization. Plant Physiol. 1996;110: 23-33.

919 28. Sanderfoot AA., Lazarowitz SG. Getting it together in plant virus movement: cooperative 920 interactions between bipartite geminivirus movement proteins. Trends Cell Biol. 1996;6: 353-358.

921 29. Ward BM, Medville E, Lazarowitz SG, Turgeon R. The geminivirus BL1 movement protein 922 is associated with endoplasmic reticu- lum-derived tubules in developing phloem cells. J Virol. $9231997 ; 71: 3726-3733$.

924 30. Zhang SC, Wege C, Jeske H. Movement proteins (BC1 and BV1) of Abutilon mosaic 925 geminivirus are cotransported in and between cells of sink but not of source leaves as detected by 926 green fluorescent protein tagging. Virology. 2001;290: 249-260.

927 31. Aberle HJ, Rütz ML, Karayavuz M, Frischmuth S, Wege C, Hülser D, et al. Localizing BC1 928 movement proteins of Abutilon mosaic geminivirus in yeasts by subcellular fractionation and 929 freeze-fracture immunolabelling. Arch Virol. 2002;147:103-107.

930 32. Frischmuth S, Wege C, Hülser D, Jeske H. The movement protein BC1 promotes redirection 931 of the nuclear shuttle protein BV1 of Abutilon mosaic geminivirus to the plasma membrane in 932 fission yeast. Protoplasma. 2007;230:117-123. 
933 33. Krenz B, Windeisen V, Wege C, Jeske H, Kleinow T. A plastid-targeted heat shock cognate

$93470 \mathrm{kDa}$ protein interacts with the Abutilon mosaic virus movement protein. Virology. 2010;401:6-

93517.

936 34. Krenz B, Jeske H, Kleinow T. The induction of stromule formation by a plant DNA-virus in

937 epidermal leaf tissues suggests a novel intra- and intercellular macromolecular trafficking route.

938 Front Plant Sci. 2012;3: 291.

939 35. Lewis JD, Lazarowitz SG. Arabidopsis synaptotagmin SYTA regulates endocytosis and virus

940 movement protein cell-to-cell transport. Proc Natl Acad Sci, USA. 2010;107: 2491-2496.

941 36. Krapp S, Schuy C, Greiner E, Stephan I, Alberter B, Funk C, et al. Begomoviral movement

942 protein effects in human and plant cells: towards new potential interaction partners. Viruses.

$943 \quad 2017 ; 9: 334$.

944 37. Yazaki J, Galli M, Kim AY, Nito K, Aleman F, Chang KN, et al. Mapping transcription factor

945 interactome networks using HaloTag protein arrays. Proc Natl Acad Sci, USA. 2016;113: e4238-

$946 \quad 47$

947 38. Alcaide-Loridan C, Jupin I. Ubiquitin and plant viruses, let's play together! Plant Physiol. $948 \quad 2012 ; 160: 72-82$.

949 39. Conchon S, Cao X, Barlowe C, Pelham HR. Gotlp and Sft2p: membrane proteins involved in 950 traffic to the Golgi complex. EMBO J. 1999;18: 3934-3946.

951 40. Jung JJ, Inamdar SM, Tiwari A, Choudhury A. Regulation of intracellular membrane 952 trafficking and cell dynamics by syntaxin-6. Biosci Rep. 2012;32: 383-391.

953 41. Drakakaki G, van de Ven W, Pan S, Miao Y, Wang J, Keinath NF, et al. Isolation and proteomic 954 analysis of the SYP61 compartment reveal its role in exocytic trafficking in Arabidopsis. Cell Res. 955 2012;22: 413-424. 
42. Zhourar J, Rojo E, Bassham DC. AtVPS45 Is a Positive Regulator of the SYP41/SYP61/VTI12

957 SNARE Complex Involved in Trafficking of Vacuolar Cargo. Plant Physiol. 2009;149: 1668-

9581678.

959 43. Hachez C, Laloux T, Reinhardt H, Cavez D, Degand H, Grefen C, et al. Arabidopsis SNAREs

960 SYP61 and SYP121 coordinate the trafficking of plasma membrane aquaporin PIP2;7 to modulate

961 the cell membrane water permeability. Plant Cell. 2014;26: 3132-3147.

962 44. Reverter M, Rentero C, Garcia-Melero A, Hoque M, Vilà de Muga S, Alvarez-Guaita, A et al.

963 Cholesterol regulates Syntaxin 6 trafficking at trans-Golgi network endosomal boundaries. Cell 964 Rep. 2014;7: 883-97

965 45. Robatzek S, Chinchilla D, Boller T. Ligand-induced endocytosis of the pattern recognition 966 receptor FLS2 in Arabidopsis. Genes Dev. 2006;20: 537-542.

967 46. Nelson BK, Cai X, Nebenfuhr A. A multicolored set of in vivo organelle markers for co968 localization studies in Arabidopsis and other plants. Plant J. 2007;51: 1126-1136.

969 47. Haupt S, Cowan GH, Ziegler A, Roberts AG, Oparka KL, Torrance L. Two Plant-Viral

970 Movement Proteins Traffic in the Endocytic Recycling Pathway. Plant Cell. 2005;17: 164-181.

971 48. Calil IP, Quadros IPS, Araújo TC, Duarte CEM, Gouveia-Mageste BC, Silva JCF, et al. A

972 WW Domain-Containing Protein Forms Immune Nuclear Bodies against Begomoviruses. Mol

973 Plant. 2018;11: 1449-1465.

974 49. Wang T, Li L, Hong W. SNARE proteins in membrane trafficking. Traffic. 2017;18:767-775.

975 50. Zhourar, J., Rojo, E., and Bassham, D.C. (2009). AtVPS45 Is a Positive Regulator of the 976 SYP41/SYP61/VTI12 SNARE Complex Involved in Trafficking of Vacuolar Cargo. Plant 977 Physiol. 149: 1668-1678.

978 51. Jahn R, Scheller RH. SNAREs_Engines for membrane fusion. Nat Rev Mol Cell Biol. 2006; 7: $979 \quad 631-643$. 
980 52. Edgar RC. MUSCLE: multiple sequence alignment with high accuracy and high throughput.

$981 \quad$ Nucleic Acids Res. 2004;32(5): 1792-1797.

982 53. Ronquist F, Huelsenbeck JP. MrBayes 3: Bayesian phylogenetic inference under mixed 983 models. Bioinformatics. 2003;19: 1572-1574.

984 54. Brandes U. A faster algorithm for betweenness centrality. J Mathem Sociol. 2001;25: 163-177.

985 55. Freeman LC. Centrality in social networks conceptual clarification. Social Networks. 1978;1:

$986 \quad 215-239$.

987 56. Hage P, Harary F. Eccentricity and centrality in networks. Social Networks. 1995;17: 57-63.

988 57. Falcon S, Gentleman R. Using GOstats to test gene lists for GO term association. 989 Bioinformatics. 2007;23: 257-258.

990 58. Zhang X, Henriques R, Lin SS, Niu QW, Chua NH. Agrobacterium-mediated transformation 991 of Arabidopsis thaliana using the floral-dip method. Nat Protoc. 2006;1: 641-646.

992 59. Santos AA, Carvalho CM, Florentino LH, Ramos JJO, Fontes EPB. Conserved threonine 993 residues within the A-loop of the receptor NIK differentially regulate the kinase function required 994 for antiviral signaling. PLoS ONE. 2009;4: e5781.

995 60. Rocha CS, Santos AA, Machado JPB, Fontes EPB. The ribosomal protein L10/QM-like protein 996 is a component of the NIK-mediated antiviral signaling. Virology. 2008;380: 165-169.

997 61. Dellaporta SL, Wood J, Hicks JB. 1983. A plant DNA minipreparation: version II. Plant Mol 998 Biol Rep. 1983;1: 19-23.

999 62. Rojas MR, Gilbertson RL, Russel DR, Maxwell DP. Use of degenerate primers in the 1000 polymerase chain reaction to detect whitefly-transmitted geminiviruses. Plant Dis. 1993;77: 3401001347. 
63. Alves MS, Reis PAB, Dadalto SP, Faria JAQA, Fontes EPB, Fietto LG. A Novel Transcription

1003

1004

1005

1006

1007

1008

1009

1010

1011

1012

1013

1014

1015

1016

1017 1018 functionally similar hubs.

1019

1020

1021

1022

1023

1024

Figure Legends

\section{Fig 1. MP-NSP-Arabidopsis protein-protein interactions (PPI) network}

\section{integrated into Arabidopsis interactome.}

The network displays a firework topology, which was assembled by the Cytoscape software. The viral proteins, MP and NSP, are represented in red. The NSP-specifically interacting proteins are in dark orange and connecting proteins from Arabidopsis in light orange. The MP-specifically interacting proteins are indicated in light yellow to which interacting dark yellow proteins converge. Arabidopsis proteins that interact with both MP and NSP are shown in green, forming hubs of convergent blue proteins. Gray proteins represent connecting points between two

\section{Fig 2. NISP, but not AT2G18860, interacts with NSP via the Syntaxin-6 domain.}

(A) Schematic representation of NISP and AT2G18860 sequence alignment. The syntaxin-6 domain is indicated in green and the transmembrane segment, in red. The identity of sequence and positions of amino acid residues are indicated. Sequence alignment was performed with CLUSTAL OMEGA. (B) NISP and NSP interact in yeast. NISP and NSP fused to the activation 
1025 domain (AD), and binding domain (BD) of Gal4 and also in combination with empty vectors were

1026 expressed in yeast. The interactions between the tested proteins were monitored by histidine

1027 prototrophy in medium supplemented with $3-\mathrm{AT}$ for 4 days at $28^{\circ} \mathrm{C}$. (C) AT2G18860 failed to

1028 interact with NSP in yeast. AT2G18860 and NSP fused to both Gal4AD and BD were expressed

1029 in yeast, and possible interactions were assayed in all combinations. Co-expressed NSP-BD and

1030 NISP-AD were used as a positive control. (D-F). Mapping the NSP-binding domain on NISP.

1031 Yeast cells expressing the indicated truncated version of NISP and NSP fusions were plated on

1032 selective medium lacking leucine, tryptophan, and histidine and supplemented with 2.5 mM 3-AT

1033 and grown for 4 days at $28^{\circ} \mathrm{C}$. The truncated versions of NISP are delimited by the indicated

1034 position of the amino acid residues in the full-length protein. Protein interaction was only observed

1035 by co-expressing AD-NISP ${ }^{1-107}$ and BD-NSP.

1036

1037 Fig 3. Subcellular localization of NISP.

1038 (A) The NISP-GFP distribution in intracellular vesicles. N. benthamiana leaves were infiltrated

1039 with $A$. tumefaciens carrying a DNA construct expressing NISP-GFP under the control of 35S

1040 promoter. Confocal images from three independent experiments were taken 36 hours post-

1041 infiltration. Approximately 200 cells were examined. Scale bars, $10 \mu \mathrm{m}$ and $20 \mu \mathrm{m}$. (B) Confocal

1042 images of NISP-GFP transiently expressed under the control of the endogenous promoter. Scale

1043 bars, $10 \mu \mathrm{m}$ and $20 \mu \mathrm{m}$. (C) Co-expression of the ER-associated ER-mCherry molecular marker

1044 and NISP-GFP in N. benthamiana leaves. N. benthamiana leaf cells expressing the indicated

1045 fusion proteins were examined by confocal microscopy 2 days after infiltration. The asterisks

1046 indicate the localization of the amplified insets, and the arrows indicate the merged pattern of GFP-

1047 associated vesicles in C, D, E, F, G, H. The confocal images are representative samples from three 
1048 independent experiments. Scale bars, $10 \mu \mathrm{m}$. (D) Co-expression of the Golgi-associated G1049 mCherry molecular marker and NISP-GFP in N. benthamiana leaves 2 days after agro-infiltration. 1050 Scale bars, $20 \mu \mathrm{m}$. (E) Co-expression of the tonoplast-associated vac-mCherry molecular marker 1051 and NISP-GFP in N. benthamiana leaves 2 days after agro-infiltration. Scale bars, $20 \mu \mathrm{m}$. (F) Co1052 expression of the plasma membrane-associated FLS2-mCherry and NISP-GFP in N. benthamiana 1053 leaves 2 days after agro-infiltration. Scale bars, $20 \mu \mathrm{m}$. (G) Co-expression of endosome-associated 1054 SYTA-mCherry and NISP-GFP in N. benthamiana leaves 2 days after agro-infiltration. Scale bars, $105510 \mu \mathrm{m}$. (H) Co-labeling of vesicles with NISP-GFP and FM4-64 in epidermal cells of $N$. benthamiana. Images were taken after 2 days post infiltration of Agrobacterium carrying a NISP-

1057 GFP expression vector and $1 \mathrm{~h}$ after infiltration of FM4-64. Scale bars, $20 \mu \mathrm{m}$.

1058

1059 Fig 4. NISP interacts with NSP in vivo.

1060 (A) NISP interacts with NSP in vesicle-like compartments, as judged by BiFC. N. benthamiana 1061 leaf cells expressing NISP and NSP fused to the C-terminus (cYFP) or N-terminus (nYFP) of YFP, 1062 or in combination with the empty vectors, were observed by confocal microscopy 3 days after 1063 infiltration. Scale bars, $10 \mu \mathrm{m}$. The experiment was repeated at least four times with identical 1064 results. (B) NISP-NSP complex-containing vesicles are stained by FM4-64. N. benthamiana leaves 1065 were agroinfiltrated with nYFP-NISP- and cYFP-NSP-expressing DNA constructs. Images were 1066 taken 2 days post agroinfiltration and $30 \mathrm{~min}$ after infiltration of FM4-64. Arrows indicate 1067 examples of vesicle-associated reconstituted fluorescent signals that were co-stained by FM4-64. 1068 Scale bars, $10 \mu \mathrm{m}$. The experiment was repeated three times with similar results. (C) NISP interacts 1069 with NSP in planta. Whole cell protein extracts from N. benthamiana leaves expressing transiently 1070 NISP-GFP and NSP-6HA were used for co-immunoprecipitation assays using an anti-GFP serum. 
1071 Input and IP show the levels of the expressed proteins NSP-6HA, NISP-GFP, and GFP. Anti-HA

1072 antibody was used to detect NSP-6HA from the immunoprecipitated complex. GFP was used as a

1073 negative control. Molecular mass markers are given on the left in kilodaltons. The experiment was

1074 repeated twice. (D) In vivo interaction of NISP with NSP. Co-immunoprecipitation was performed

1075 as described in C, except that the tags of the transiently expressed proteins were switched to NSP-

1076 GFP and NISP-6HA. The experiment was repeated twice.

\section{Fig 5. NISP displays a pro-begomoviral function.}

1079 (A) Transcript accumulation of NISP in transgenic lines. NISP transcript levels in three 1080 independently complemented transgenic lines (nisp-1/NISP-1-3), three independently transformed 1081 NISP-overexpressing lines (AtNISP1-3), Col-0 and nisp-1 were determined by qRT-PCR. Gene 1082 expression was calculated using the $2^{-\Delta \mathrm{Ct}}$ method, and actin was used as an endogenous control.

1083 Error bars represent $95 \%$ confidence intervals based on replicated samples $(\mathrm{n}=3)$ from three 1084 independent experiments. (B) NISP-GFP protein accumulation in transgenic lines. Whole cell 1085 protein extracts from the complemented transgenic lines (nisp-1/NISP), NISP-overexpressing lines 1086 (AtNISP), Col-0 and nisp- 1 were separated by SDS-PAGE and immunoblotted using an anti-GFP 1087 serum. (C) AT2G18860 transcript accumulation in transgenic lines. The transcript levels of 1088 AT2G18860 in complemented transgenic lines (at2g18860-1/AT2G18860-1-3), AT2G188601089 overexpressing lines (AT2G18860-1-3), Col-0 and at2g18860-1 were quantified by qRT-PCR and 1090 normalized to the actin as an endogenous control. Error bars indicate 95\% confidence intervals 1091 based on replicated samples $(n=3)$ from three independent experiments. (D) AT2G18860-GFP 1092 protein accumulation in transgenic lines. Total protein extracts from complemented transgenic 1093 lines (at2g18860-1/AT2G18860), AT2G18860-overexpressing lines (AT2G18860), Col-0 and 

(dpi). The figure shows representative samples of mock-inoculated and CabLCV -infected plants. The genotypes are indicated in the figure and are the same as specified in A-D. (F) The onset of infection is delayed in nisp-1 knockout line. Ecotype Col-0, nisp-1, at2g18860-1, nisp-1/NISP complemented lines and AtNISP-overexpressing lines (as in a-d) were infected with CabLCV DNA, and the course of infection was monitored as the percent of systemically infected plants at different dpi. (G) Absolute quantification of CabLCV genomic units in infected lines at 21 dpi. The genotypes are the same as specified in a-d. Error bars indicate 95\% confidence intervals based on replicated samples from three independent experiments. Different letters indicate significative 1104 differences among the groups (Duncan test, $\mathrm{p}<0,05, \mathrm{n}=3$ ).

\section{formation.}

(A) BIFC assay showing the interaction between NISP and NIG in vesicles of $N$. benthamiana leaf cells. N. benthamiana leaves transiently expressing NISP and NIG fused to the C-terminus (cYFP) or N-terminus (nYFP) of YFP were examined by confocal microscopy 3 days after infiltration.

1111 Scale bars, $10 \mu \mathrm{m}$ and $20 \mu \mathrm{m}$. The experiment was repeated three times with similar results. (B)

1112 NISP-NIG complex-containing vesicles are stained by FM4-64. N. benthamiana leaves were 1113 agroinfiltrated with nYFP-NIG- and cYFP-NISP-expressing DNA constructs. Images were taken 11142 days post infiltration and $1 \mathrm{~h}$ after infiltration of FM4-64. Arrows indicate examples of vesicle1115 associated reconstituted fluorescent signals that were co-stained by FM4-64 and the asterisk, the 1116 region of the amplified inset. Scale bars, $10 \mu \mathrm{m}$. The experiment was repeated three times with 
1117 identical results. (C) In vivo interaction of NISP and NIG. Total protein extracts from $N$.

1118 benthamiana expressing NISP-GFP and NIG-6HA were used for co-immunoprecipitation assays

1119 using anti-GFP. Input and IP show the levels of the expressed proteins NISP-GFP and NIG-6HA.

1120 Anti-HA was used to detect NIG-6HA from the immunoprecipitated complex. GFP was used as

1121 an unrelated protein. The experiment was repeated twice with identical results. (D) NISP-NIG

1122 complex formation in the presence of viral NSP. The Co-IP assay was performed as described in

1123 A, except that co-expressed NSP-GST was included in the assay. NSP-GST was detected by

1124 immunoprecipitating it from infiltrated leaves and immunoblotting with anti-GST. The experiment

1125 was repeated twice with identical results. (E) Viral NSP enhances the interaction between NISP

1126 and NIG. NIG levels in the immunoprecipitated complex in the presence and absence of viral NSP

1127 were quantified using the Band Analysis tools of the ImageLab software (Bio-Rad). The signal

1128 values were normalized using the IP NISP-GFP band, revealed in the same blot as the Co-IP band.

1129 A.U. denotes arbitrary units.

\section{Fig 7. NISP complexes with vDNA in vivo.}

1132 (A) NISP-GFP accumulation in overexpressing lines. Whole cell protein extracts from the NISP-

1133 GFP-overexpressing lines, as indicated in the figure, and Col-0 were separated by SDS-PAGE and

1134 immunoblotted using an anti-GFP serum. (B) NISP binds to viral DNA-B in vivo. NISP-GFP-

1135 overexpressing lines (AtNISP-1, AtNISP-2, and AtNISP-3), and Col-0 were inoculated with

1136 infectious CabLCV clones prior to the ChIP assay, which was performed with leaf samples of the

1137 indicated genotypes using anti-GFP. The infected plants were confirmed by PCR of input DNA

1138 from inoculated plants using CabLCV DNA-B-specific primers, which amplifies a 770-bp

1139 fragment (F1/R1; S10b Fig). IN denotes infected leaves and UN, uninfected leaves. (C) ChIP- 
1140 qPCR assay of leaves from AtNISP-GFP transgenic lines and Col-0. The samples were

1141 immunoprecipitated with an anti-GFP antibody and ChIPed DNA was quantified by qPCR using

1142 the DNA-B-specific primers F3/R3 (S10b Fig). Data were normalized relative to the input of each

1143 sample and are expressed as the percentage of input. (D) ChIP-qPCR assay using a different set of

1144 DNA-B-specific primers (F2/R2) and samples from the indicated genotypes. The ChIP-qPCR was

1145 performed as described in C. (E) NISP binds to viral DNA-A in vivo. NISP-GFP-overexpressing

1146 lines (AtNISP-1, AtNISP-2 and AtNISP-3), and Col-0 were inoculated with infectious CabLCV

1147 clones prior to the ChIP assay, which was performed with leaf samples of the indicated genotypes

1148 using anti-GFP. The infected plants were confirmed by PCR of input DNA from inoculated plants

1149 using CabLCV DNA-A-specific primers, which amplifies a 389-bp fragment (F1/R1; S10a Fig).

1150 IN denotes infected leaves and UN, uninfected leaves. (F) ChIP-qPCR assay using a set of DNA-

1151 A-specific primers (F2/R2, S10a Fig) and samples from the indicated genotypes. The ChIP-qPCR

1152 was performed as described in C. The above experiments were repeated three times with similar 1153 results.

1155 Supporting information

1156 S1 Table- Protein-protein interactions between the viral proteins and the 1157 Arabidopsis proteins.

1159 S2 Table- Enriched GO terms in three categories, Biological Process, Molecular 1160 Function, or Cellular Component ontology 
1162 S3 Table. List of primers used for cloning, PCR, RT-qPCR and qPCR

1164 S4 Table. Expression of kanamycin resistance in the T1 generation of transgenic 1165 Arabidopsis plants.

1167 S1 Video/Movie -Vesicle-associated localization of NISP

1169 S1 Fig. Identification of MP- and NSP-interacting proteins.

1170 In vitro synthesized 3xHA-NSP and 3xHA-MP. In vitro translated HA-NSP and HA-MP were

1171 electrophoresed by SDS-PAGE and immunoblotted using an anti-HA antibody. (B) Protein

1172 interaction between in situ synthesized Arabidopsis hallo-proteins and 3xHA-MP or 3xHA-NSP.

1173 The AtNAPPA02 high-density array containing 4600 Arabidopsis ORFS spotted in duplicates was

1174 probed with $3 \times$ HA-MP or $3 \times$ HA-NSP, and candidate interactors were detected with an anti-HA

1175 antibody. The bright spots are signals from candidate interactors.

1177 S2 Fig. A network of NSP- and MP-directly interacting Arabidopsis proteins.

1178 The network was assembled by the Cytoscape software. The viral proteins are indicated in red,

1179 MP-specifically interacting proteins in yellow, NSP-specifically interacting proteins in orange and 1180 proteins that associate with both MP and NSP are indicated in green. 
1183 (A) Phylogenetic tree of syntaxin superfamily domain-containing proteins from the species

1184 indicated. The phylogenetic tree was constructed using Bayesian inference performed with

1185 MrBayes v3.2.2 and the mixed amino acid substitution model Wag. The syntaxin-6 domain-

1186 containing proteins from Arabidopsis are indicated in blue, and NISP (AT4G30240) was clustered

1187 with plant-specific syntaxin-6 proteins forming the red clade. (B) Phylogenetic tree of N-terminal

1188 Syntaxin-6 domain-containing proteins. NISP (AT4G30240) clusters together with plant-specific

1189 N-terminal syntaxin-6 proteins, which do not harbor a typical t-SNARE domain at the C-terminus.

1190 The syntaxin-6 domain proteins from Arabidopsis are in blue. The phylogenetic tree was

1191 constructed using Bayesian inference performed with MrBayes v3.2.2 and the mixed amino acid

1192 substitution model Jones.

1194 S4 Fig. Sequence alignment of NISP and AT2G18860

1195 The N-terminal syntaxin-6 domain is indicated in red and the transmembrane segment in black.

1196 The sequence alignment was performed using CLUSTAL OMEGA. Identical amino acids are

1197 indicated with asterisks, highly conserved residues with (:) and lower conservation with (.).

1199 S5 Fig. Subcellular localization of AT2G18860

1200 AT2G18860-GFP distribution in plasma membrane-associated vesicles. $N$. benthamiana leaves

1201 were infiltrated with A. tumefaciens carrying a DNA construct expressing AT2G18860-GFP under

1202 the control of $35 \mathrm{~S}$ promoter. Confocal images were taken $36 \mathrm{~h}$ post-infiltration. Approximately

1203200 cells were examined. Scale bars, $10 \mu \mathrm{m}$.

1204

1205 S6 Fig. NISP, but not AT2G18860, expression is induced by CabLCV 
1206 NISP and AT2G18860 transcript levels were quantified by qRT-PCR in uninfected Col-0 leaves

1207 (Col-0), tungsten-inoculated Col-0 leaves (Col-0 T), and systemically infected Col-0 leaves with

1208 CabLCV (Col-0 IN). Gene expression was calculated using the $2^{-\Delta \mathrm{Ct}}$ method, and actin was used

1209 as an endogenous control. Error bars indicate 95\% confidence intervals based on replicated

1210 samples $(\mathrm{n}=3)$ from three independent experiments.

1211

1212 S7 Fig. CabLCV infection-associated symptoms in Arabidopsis genotypes at 21 days post-

1213 inoculation (dpi). The figure shows representative samples of mock-inoculated and CabLCV -

1214 infected plants. The genotypes are indicated in the figure.

1216 S8 Fig. The transgenic lines are visibly indistinguishable from the wild-type plants.

1217 Columbia (Col-0) ecotype of Arabidopsis thaliana was used as the wild-type control for phenotype

1218 comparison, and the Col-0 ecotype was used for generating almost all the transgenic plants except

1219 for the complementation test in nikl-1 and at2g18860-1 mutants. The genotypes are the same as

1220 in Fig. 5. Plants were grown in a growth chamber at $22^{\circ} \mathrm{C}$ under long-day conditions $(16 \mathrm{~h}$ light $/ 8$

$1221 \mathrm{~h}$ dark). (A) Developmental phenotypes associated with inactivation of NISP gene and

1222 overexpression of NISP-GFP in the R3 generation of transgenic lines at 45 days after germination.

1223 (B) Developmental phenotypes associated with inactivation of NISP gene and overexpression of

1224 NISP-GFP in the R3 generation of transgenic lines at 60 days after germination. (C)

1225 Developmental phenotypes associated with inactivation of AT2G18860 gene and overexpression

1226 of AT2G18860-GFP in the R2 generation of transgenic lines at 45 days after germination. (D)

1227 Developmental phenotypes associated with inactivation of AT2G18860 gene and overexpression

1228 of AT2G18860-GFP in the R2 generation of transgenic lines at 60 days after germination 
1230 S9 Fig. NSP enhances NISP-NIG complex formation.

1231 (A) NISP interacts with NIG in yeast. AD-NIG and BD-NISP fusions were expressed in yeast and 1232 interactions between the recombinant proteins were monitored by His prototrophy in selective 1233 medium (-Leu,-Trp,-His) and supplemented with $2.5 \mathrm{mM}$ 3-AT. The co-expression of PRNIG

1234 fused to BD and CSN5A fused to AD was used as a positive control. (B) Cytosolic and perinuclear 1235 distribution of NIG-GFP. N. benthamiana leaves were infiltrated with A. tumefaciens carrying 1236 NIG-GFP constructs with expression driven by the $35 \mathrm{~S}$ constitutive promoter. NIG-GFP was 1237 imaged by confocal microscopy $36 \mathrm{~h}$ after infiltration. (C) Cytosolic localization of NIG in 1238 transgenic lines. Confocal fluorescence image of Arabidopsis root cells stably transformed with 1239 35S:NIG-GFP. (D) NISP interacts with NIG in planta. Total protein extracts from N. benthamiana 1240 expressing NISP-6HA and NIG-GFP were used for co-immunoprecipitation assays using anti1241 GFP. Input and IP show the levels of the expressed proteins NISP-6HA and NIG-GFP. Anti-HA 1242 was used to detect NISP-6HA from the immunoprecipitated complex. GFP was used as an 1243 unrelated protein. The experiment was repeated three times. (E) NISP-NIG complex formation in 1244 the presence of viral NSP. The Co-IP assay was performed as described in A, except that co1245 expressed NSP-GST was included in the assay. NSP-GST was detected by immunoprecipitating 1246 it from transfected leaves and immunoblotting with anti-GST. The experiment was repeated three 1247 times. (F) The interaction of NISP and NIG is increased by the presence of viral NSP. NIG-GFP 1248 levels in the immunoprecipitated complex in the presence and absence of viral NSP were 1249 quantified using the Band Analysis tools of the ImageLab software (Bio-Rad). The signal values 1250 were normalized using the IP NIG-GFP band, revealed in the same blot as the Co-IP band. A.U. 1251 denotes arbitrary unit. 
1253 S10 Fig. NISP interacts with NIG in vivo and NSP enhances NISP-NIG complex formation.

1254 (A) BIFC assay showing the interaction between NISP and NIG in vesicles of N. benthamiana leaf 1255 cells. N. benthamiana leaves transiently expressing NISP and NIG fused to the C-terminus (cYFP) 1256 or N-terminus (nYFP) of YFP, were examined by confocal microscopy 3 days after infiltration.

1257 Scale bars, $10 \mu \mathrm{m}$ and $20 \mu \mathrm{m}$. (B) Co-expression of NSP-6HA strengthens interaction between 1258 NIG and NISP. The BiFC assay was performed by agro-infiltration of $N$. benthamiana leaves with 1259 nYFP-NIG, cYFP-NISP and vice-versus along with the expression of NSP-6HA. The reconstituted 1260 fluorescence signal was observed by confocal microscopy, 3 days after agroinfiltration. The figure 1261 displays representative samples from three independent biological repeats. Scale bars, $10 \mu \mathrm{m}$. (C) 1262 Confocal fluorescent image of NISP and NIG fused to the C-terminus (cYFP) or N-terminus 1263 (nYFP) of YFP in combination with empty vectors, as indicated in the figure.

1266 (A) Schematic representation of DNA-A. The number 1 corresponds to the 5' end of the nick site 1267 (TAATATT/AC) within the conserved nonanucleotide sequence located in the intergenic region. 1268 The ORFs are indicated with arrows. The positions of the primers used for PCR (F1/R1), and qRT1269 PCR (F2/R2) are indicated. (B) Schematic representation of DNA-B. The numbering scheme is 1270 the same as in a. The ORFs are indicated with arrows. The positions of the primers used for PCR 1271 (F1/R1), and qRT-PCR (F2/R2 and F3/R3) are indicated. 
bioRxiv preprint doi: https://doi.org/10.1101/2020.01.10.901496; this version posted June 26, 2020. The copyright holder for this preprint (which was not certified by peer review) is the author/funder. All rights reserved. No reuse allowed without permission.

\section{$1273 \quad$ Figure 1}

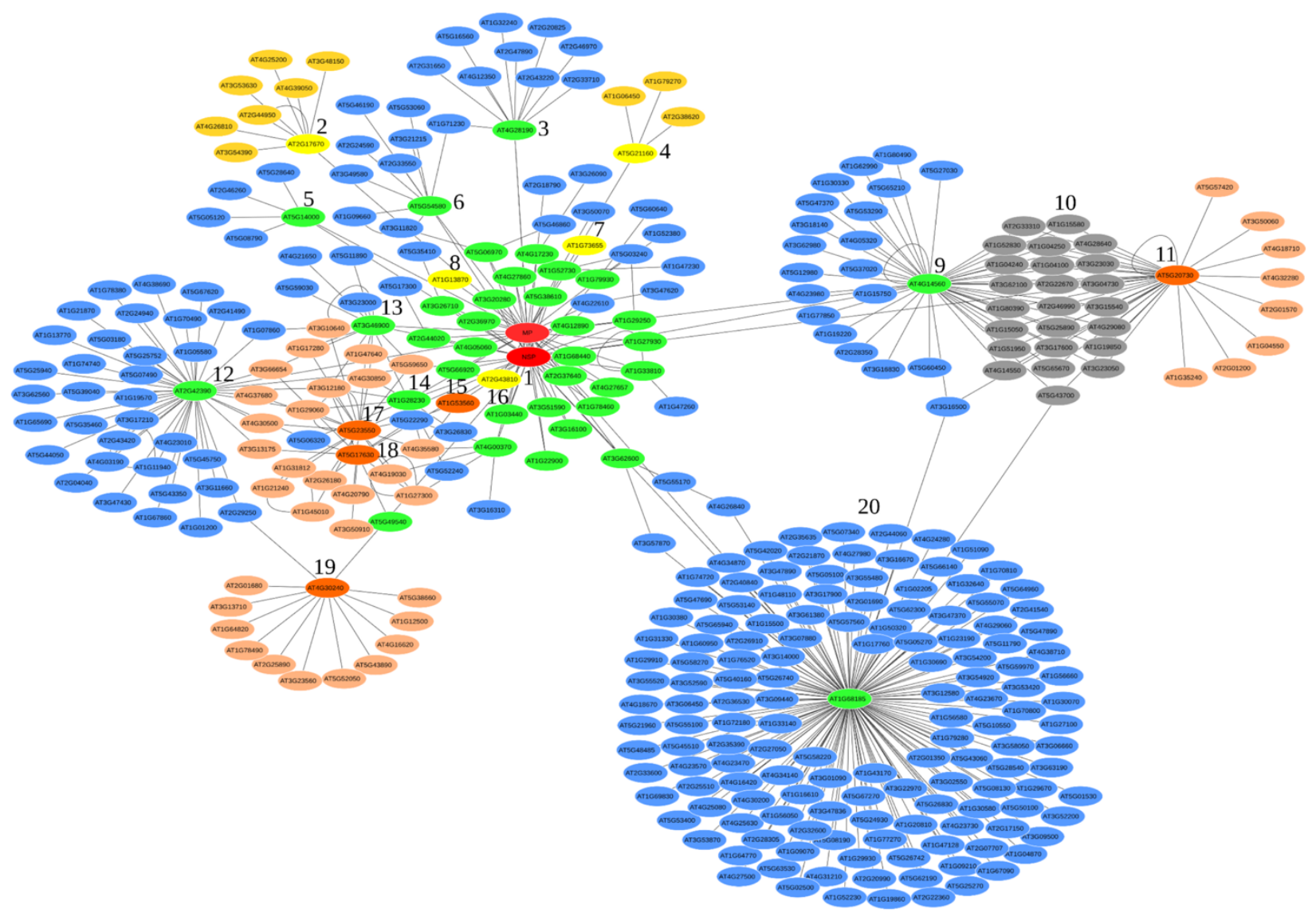


bioRxiv preprint doi: https://doi.org/10.1101/2020.01.10.901496; this version posted June 26, 2020. The copyright holder for this preprint (which was not certified by peer review) is the author/funder. All rights reserved. No reuse allowed without permission.

\section{Figure 2}

A

AT4G30240/NISP

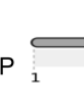

Syntaxin $6 \mathrm{~N}$ terminal

TMhelix

AT2G18860

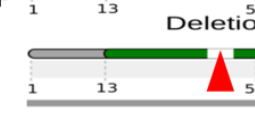

$\Lambda_{50}$

$98 \quad 150$

150

$51.76 \%$ identity

B

D

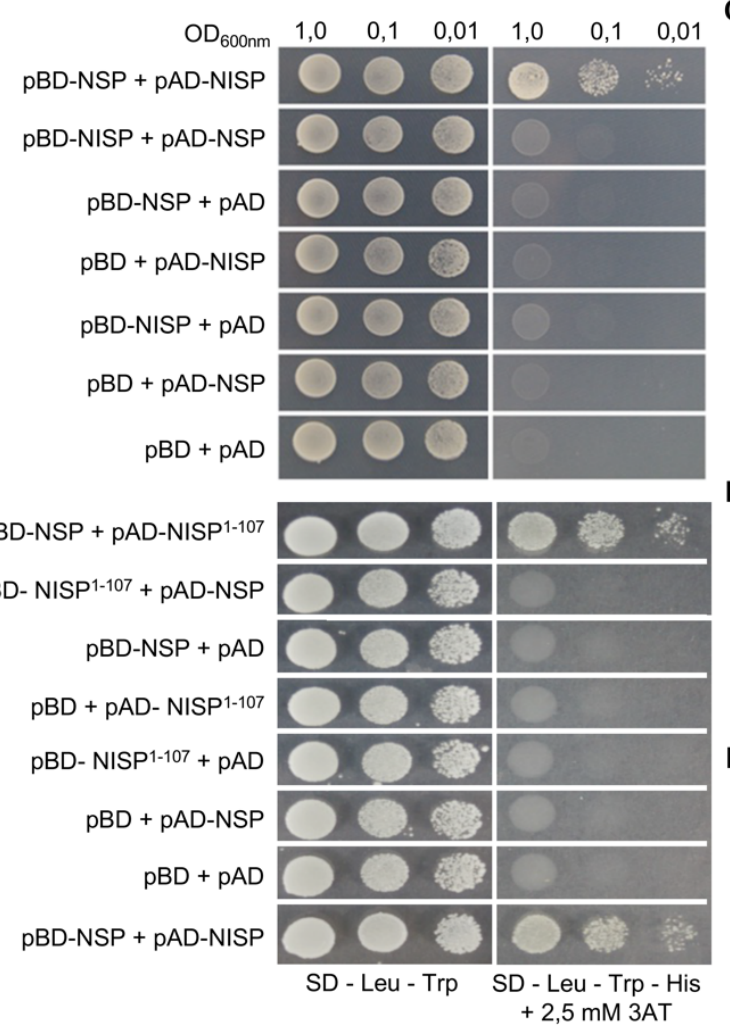

C pBD-NSP + pAD-AT2G18860 pBD-AT2G18860 + pAD-NSP pBD-NSP + pAD $p B D+p A D-A T 2 G 18860$ pBD + pAD-NSP $p B D+p A D$ pBD-NSP + pAD-NISP E pBD-NSP + pAD- NISP ${ }^{105-200}$ pBD- NISP $105-200+$ pAD-NSP pBD + pAD- NISP105-200 pBD- NISP $105-200+$ pAD F

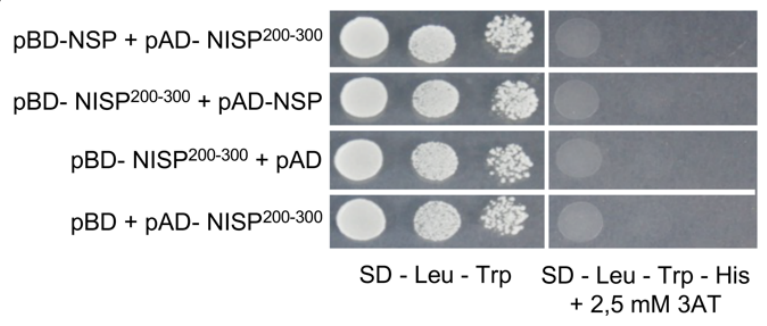




\section{Figure 3}

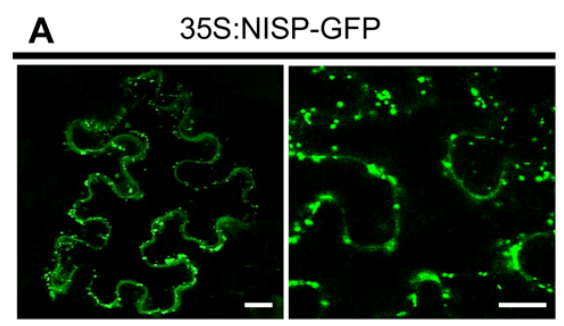

\section{B $\quad$ pNISP:NISP-GFP}

D

C

GFP

mCherry

Merge

E
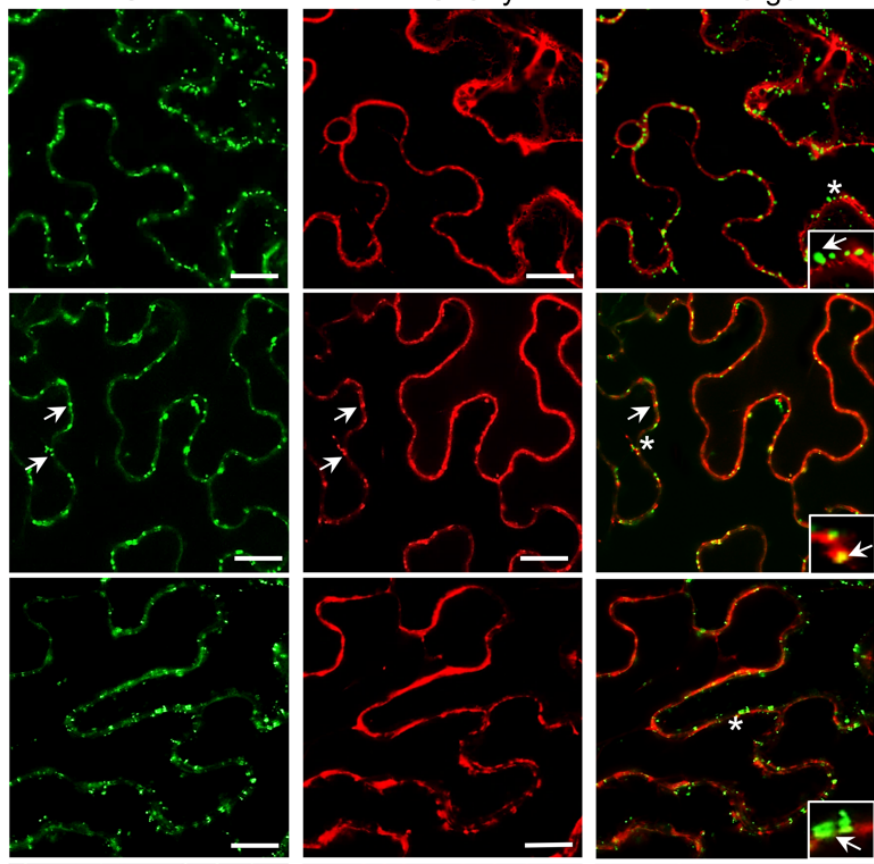

$\mathbf{F}$
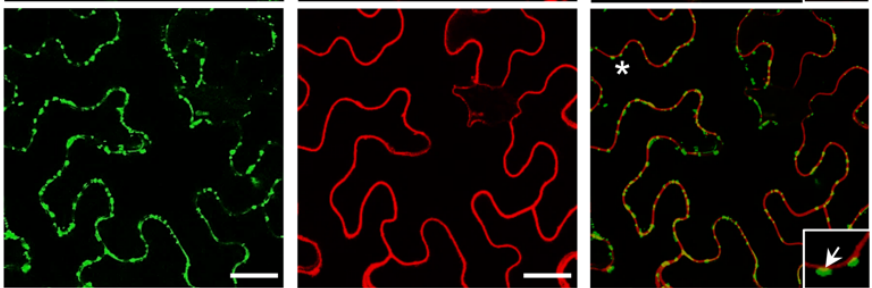

G
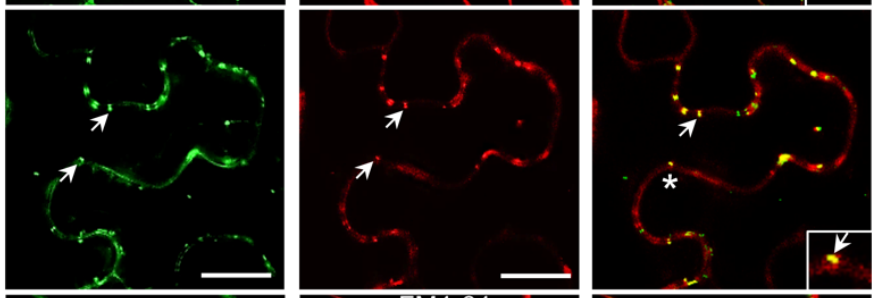

H
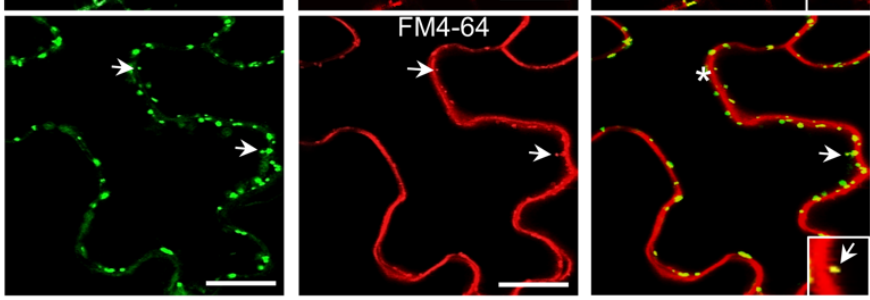
1279 Figure 4

A
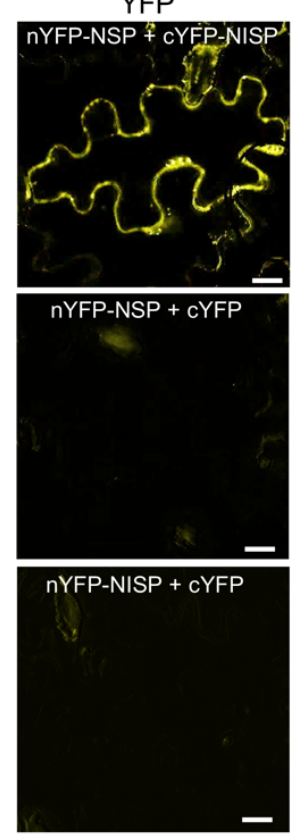

B

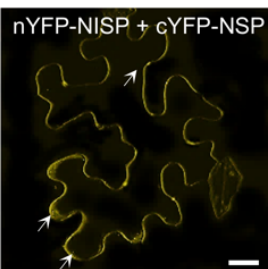

Merge
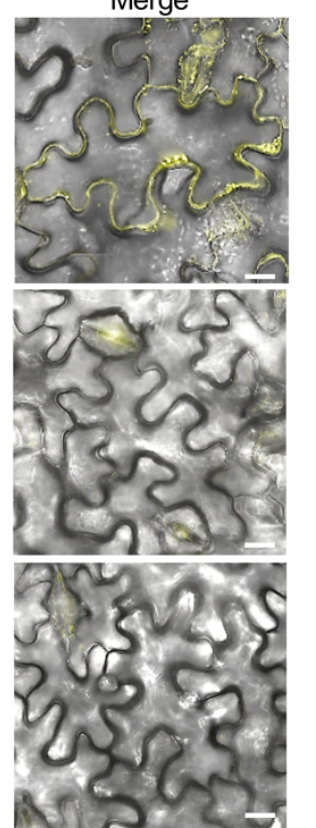

FM4-64

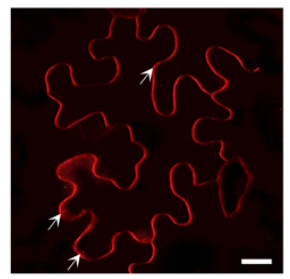

C

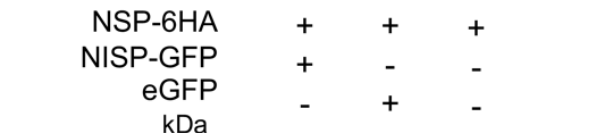

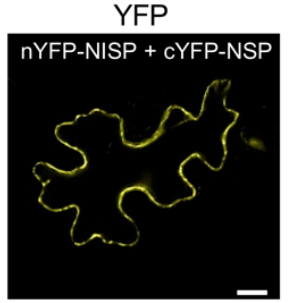
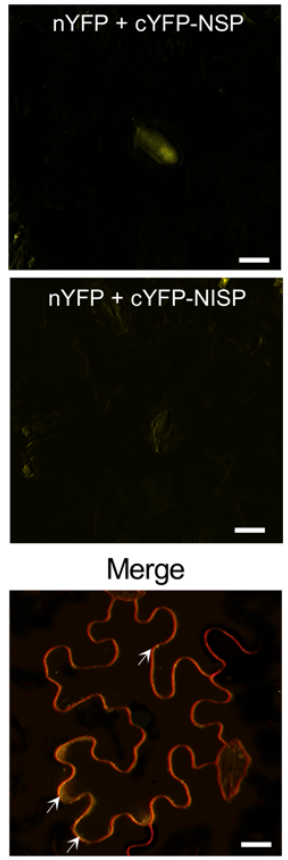

D NISP-6HA NSP-GFP

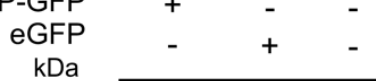
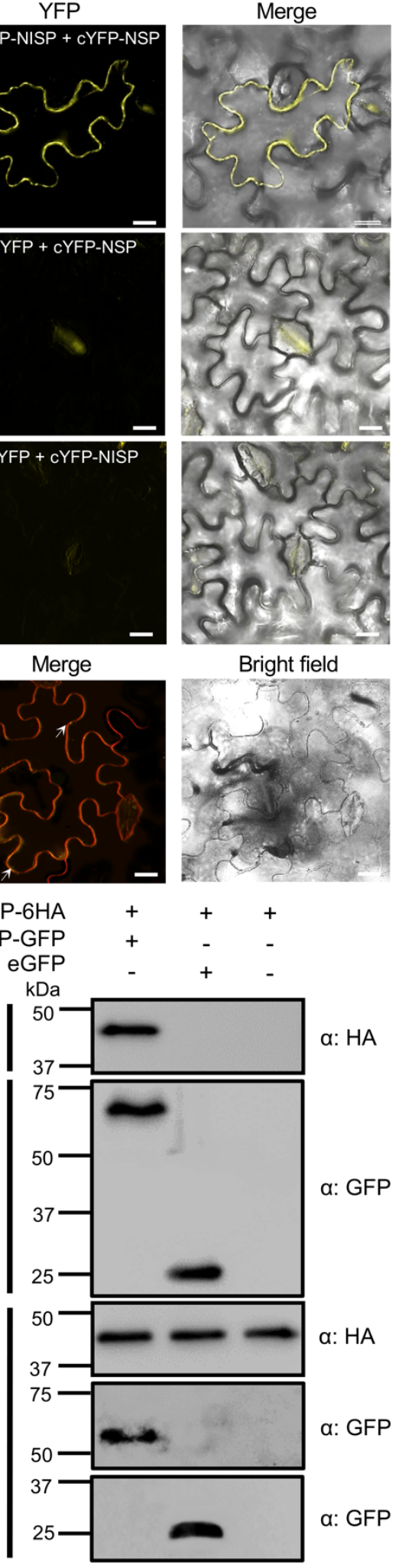

Bright field
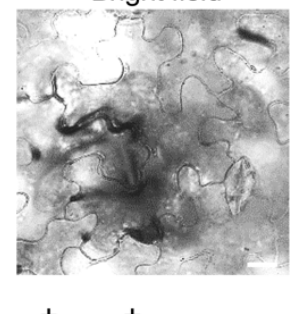$$
\text { 政 }
$$

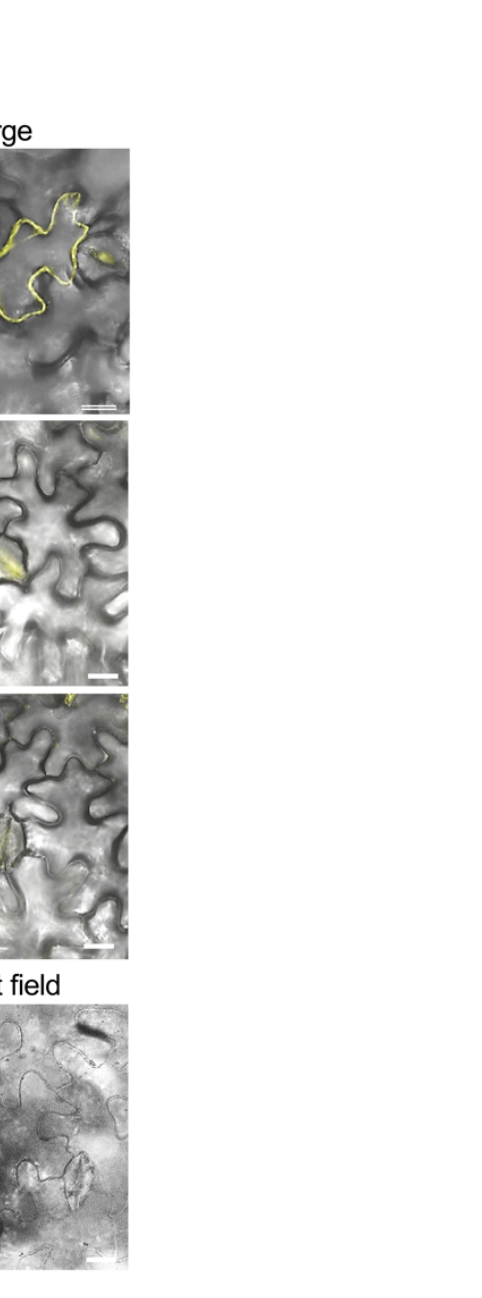




\section{Figure 5}

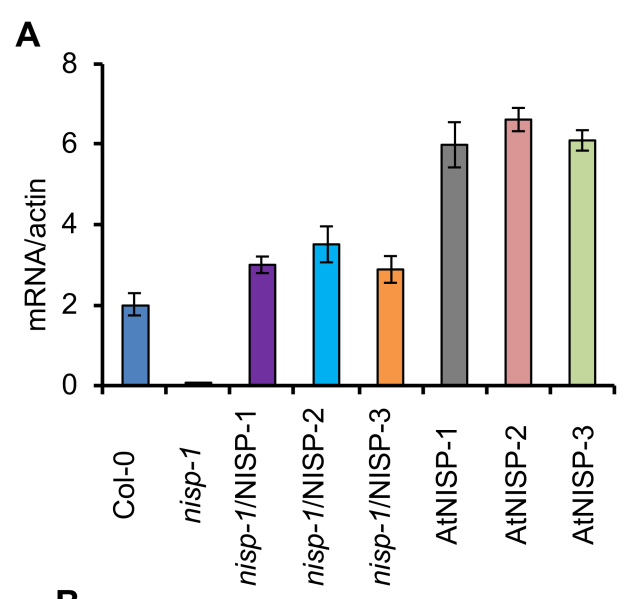

B

$\mathrm{kDa}$

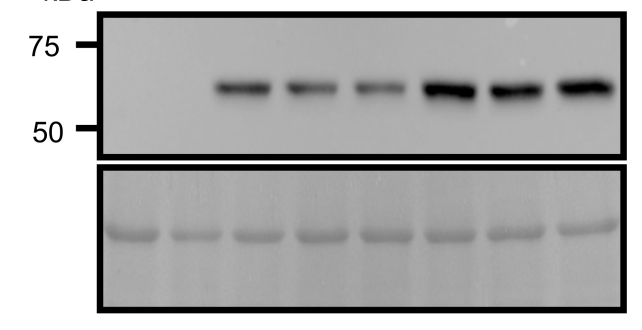

$\mathbf{E}$

$$
\text { Col-0 }
$$

nisp-1

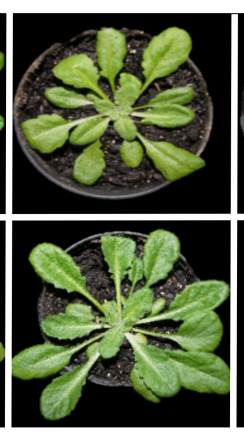

F

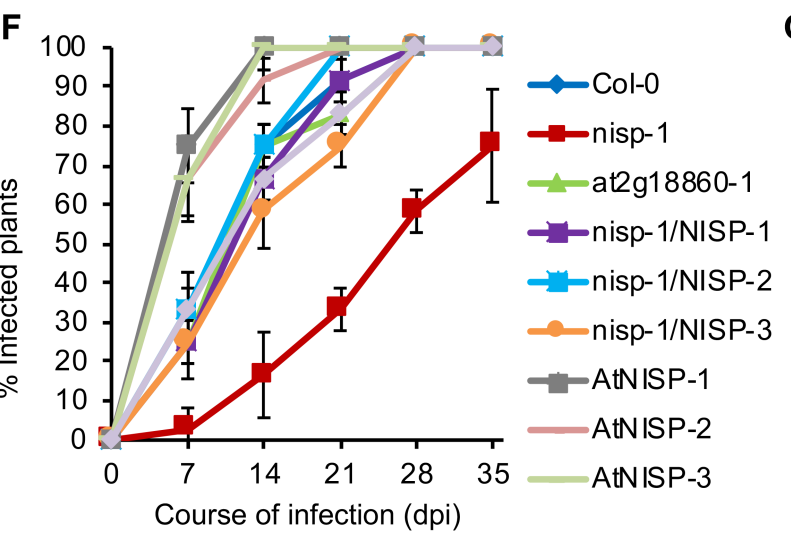

C

D

$\mathrm{kDa}$

at2g18860-1

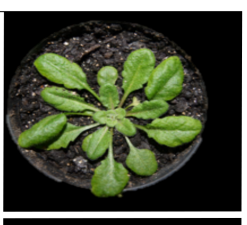

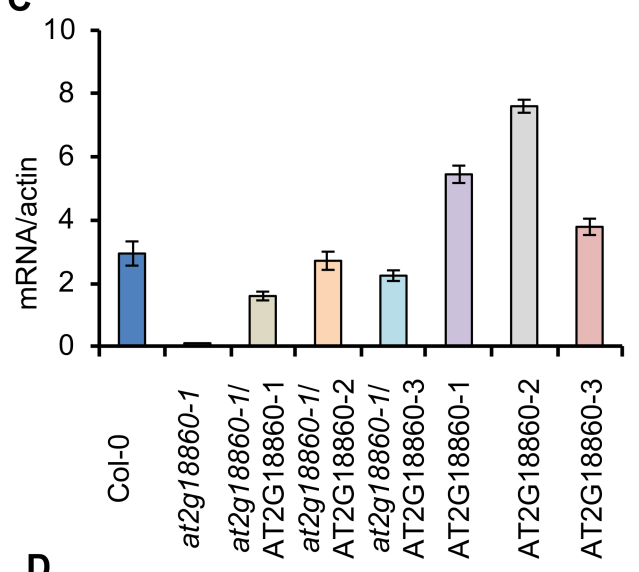

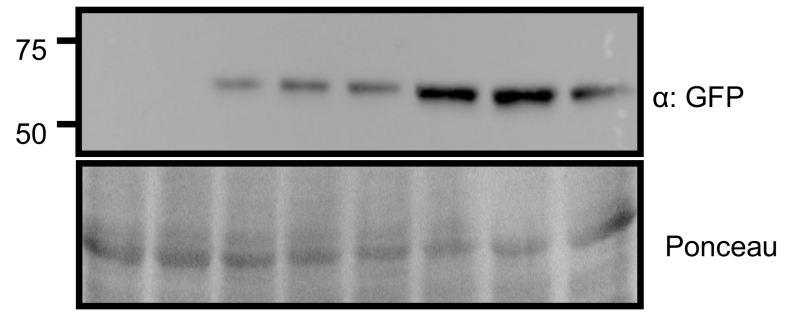

nisp-1/NISP

at2g18860-1/

AtNISP

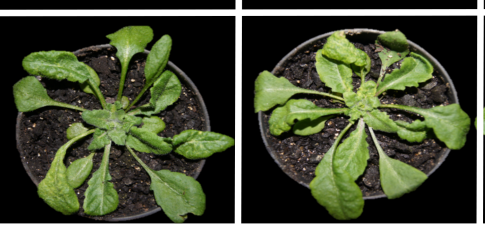

G
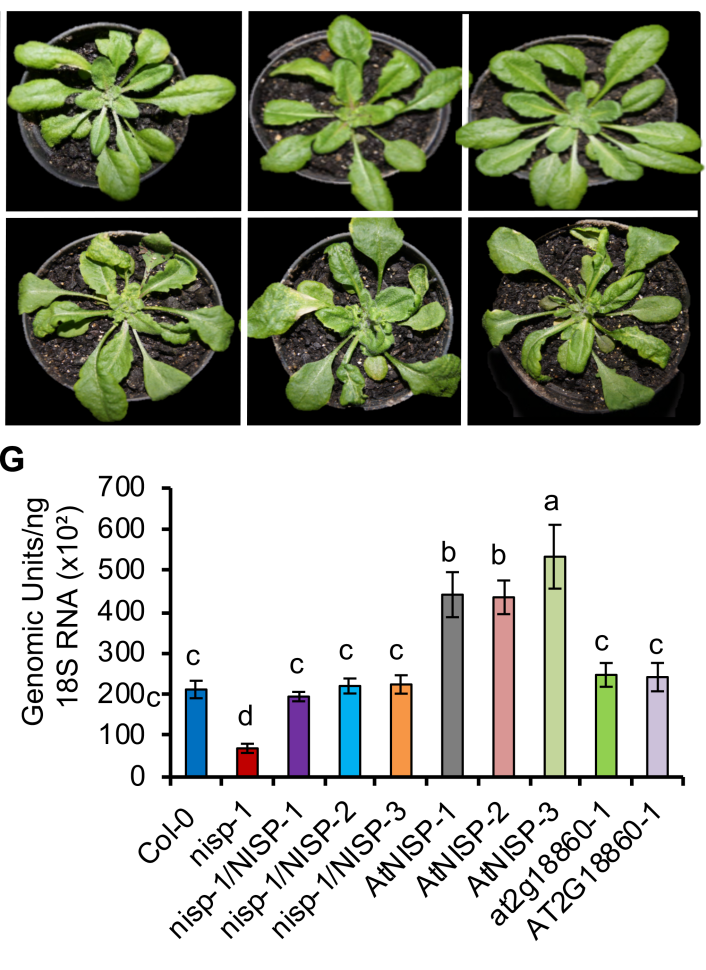


\section{Figure 6}

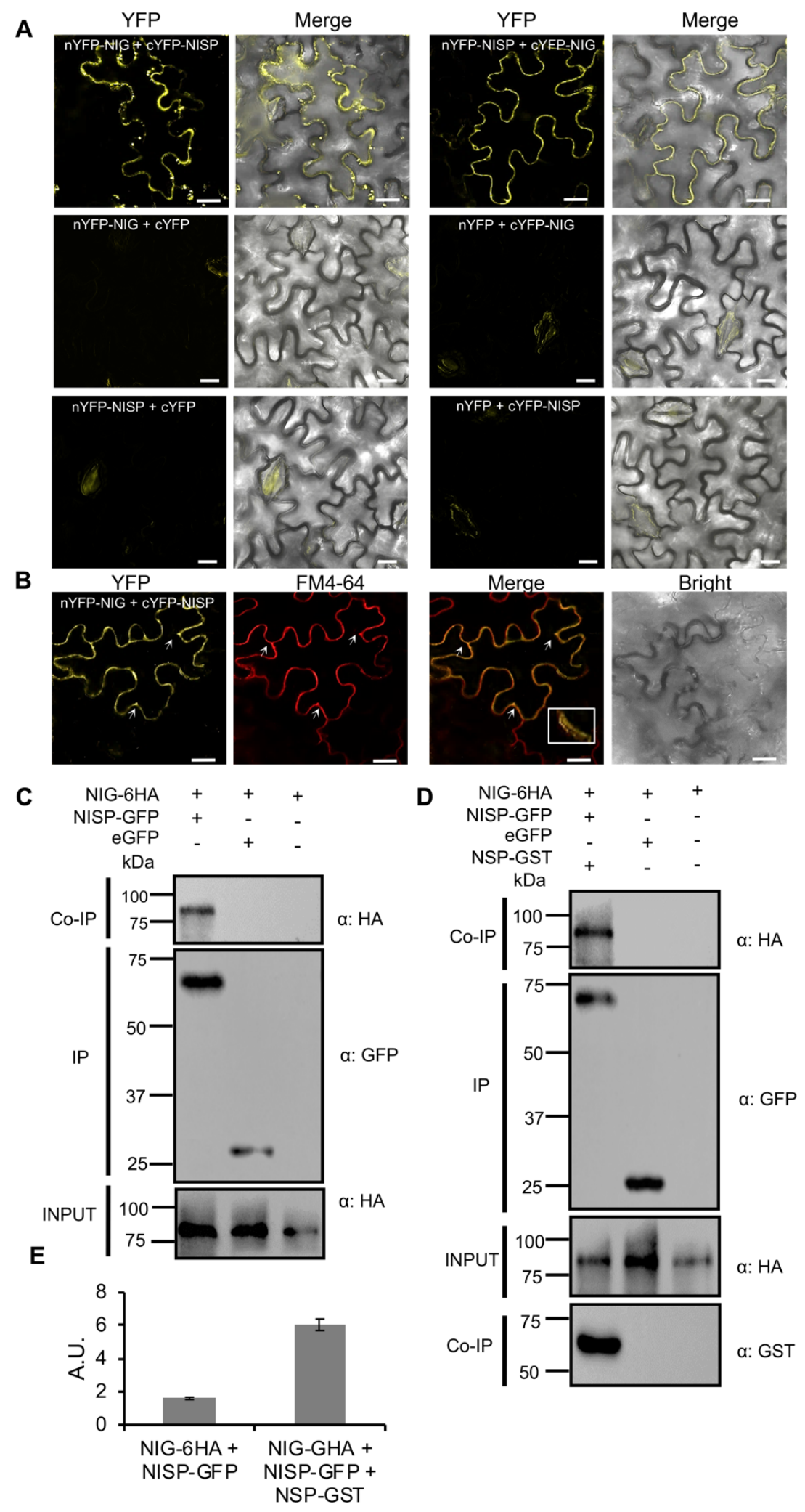




\section{$1285 \quad$ Figure 7}

A

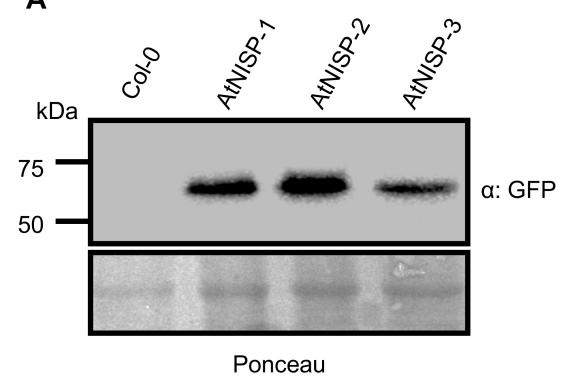

C

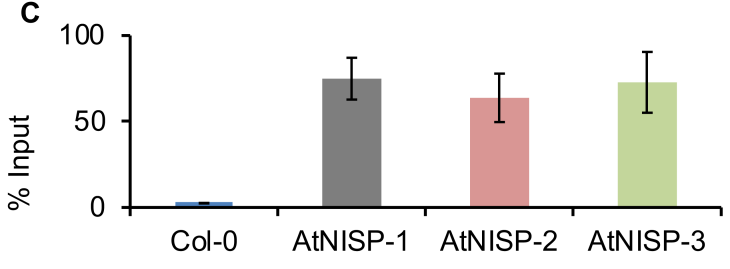

E

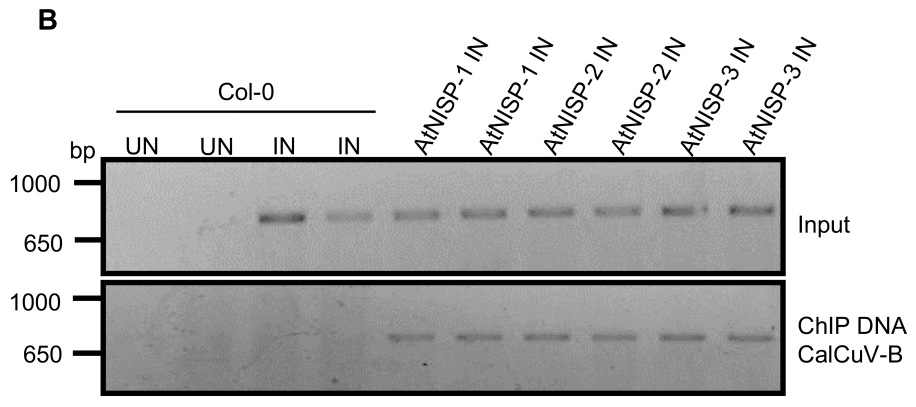

D

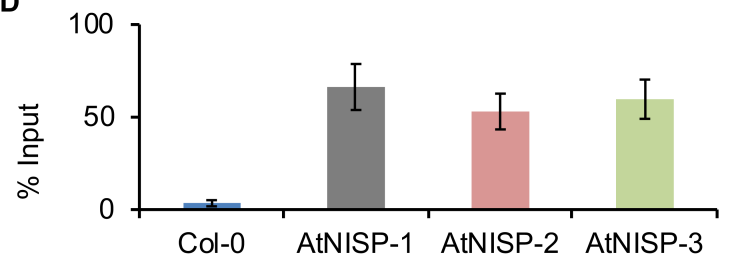

F

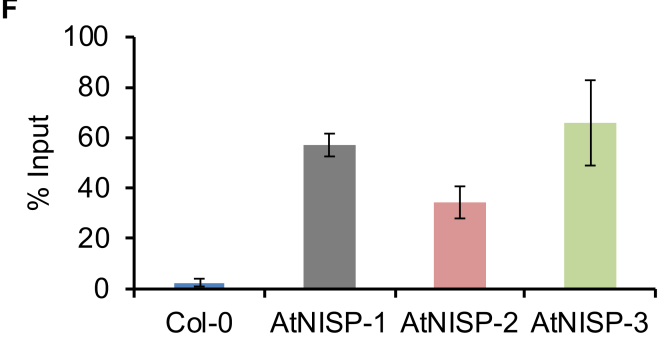

